\title{
Diffeomorfismi birazionali del piano proiettivo reale
}

\author{
Felice Ronga e Thierry Vust
}

\begin{abstract}
We study real birational transformations of the real projective plane which are diffeomorphisms. It turns out that their degree must be congruent to $1 \mathrm{mod} 4$, and that they are generated by linear automorphisms and transformations of degree 5 centred at 3 pairs of conjugated imaginary points. Our approach is inspired by recent proofs of the classical theorem of Noether and Castelnuovo that use the Sarkisov program.
\end{abstract}

Mathematics Subject Classification (2000). 14E07, 14P99.

Keywords. Real birational transformation, diffeomophism.

\section{Introduzione}

In questo lavoro si studiano trasformazioni cremoniane del piano proiettivo reale $\mathbb{P}_{\mathbb{R}}^{2}$, prive di punti base reali.

Più precisamente, sia $\mathbb{K}=\mathbb{R} \mathrm{o} \mathbb{C}$ e $\mathbb{K}\left[x_{0}, x_{1}, x_{2}\right]_{d}$ lo spazio dei polinomi omogenei di grado $d$ nelle variabili $x_{0}, x_{1}, x_{2}$, a coefficienti in $\mathbb{K}$. Una trasformazione cremoniana, o birazionale, di ordine $d$ del piano proiettivo $\mathbb{P}_{\mathbb{K}}^{2}$ è determinata da un triplo $f_{0}, f_{1}, f_{2} \in \mathbb{K}\left[x_{0}, x_{1}, x_{2}\right]_{d}$ senza fattori in comune, tale che il morfismo razionale indotto:

$$
\begin{aligned}
\varphi=\left[f_{0}, f_{1}, f_{2}\right]: \mathbb{P}_{\mathbb{K}}^{2} & \cdots \cdots \mathbb{P}_{\mathbb{K}}^{2}, \\
{\left[x_{0}, x_{1}, x_{2}\right] } & \longmapsto\left[f_{0}\left(x_{0}, x_{1}, x_{2}\right), f_{1}\left(x_{0}, x_{1}, x_{2}\right), f_{2}\left(x_{0}, x_{1}, x_{2}\right)\right]
\end{aligned}
$$

sia birazionale, il che significa che esistono aperti non vuoti, nella topologia di Zariski, $U, V \subset \mathbb{P}_{\mathbb{K}}^{2}$ tali che la restrizione di $\varphi$ a $U$ sia un isomorfismo su $V$; ne segue che esiste un inverso $\psi=\left[g_{0}, g_{1}, g_{2}\right]$, ove $g_{i} \in \mathbb{K}\left[x_{0}, x_{1}, x_{2}\right]_{d}$ (vedasi [6], [4] libro quinto, cap. II, [10] chap. VII, §4 o [1] per più ampi dettagli sulle trasformazioni cremoniane). In realtà, se si denota con $\Sigma(\varphi)$ la giacobiana di $\varphi$, cioè la curva di grado $3(d-1)$

$$
\Sigma(\varphi)=\left\{\left[x_{0}, x_{1}, x_{2}\right] \in \mathbb{P}_{\mathbb{K}}^{2} \mid \operatorname{det}\left(\frac{\partial f_{i}}{\partial x_{j}}\left(x_{0}, x_{1}, x_{2}\right)\right)=0\right\}
$$


e $U(\varphi)=\mathbb{P}_{\mathbb{K}}^{2} \backslash \Sigma(\varphi)$, allora $\varphi$ è birazionale se e solo se esiste l'inverso $\psi$ tale che $\varphi: U(\varphi) \stackrel{\simeq}{\longrightarrow} U(\psi)$.

Un modo conveniente per descrivere trasformazioni cremoniane è in termini di sistemi lineari di curve piane, a cui si prescrive di passare per dei punti $\left\{P_{1}, \ldots, P_{k}\right\} \subset$ $\mathbb{P}_{\mathbb{C}}^{2}$ con molteplicità $\lambda_{1}, \ldots, \lambda_{k}$ rispettivamente; poniamo

$$
\begin{aligned}
\mathcal{C}^{d}\left(P_{1}^{\lambda_{1}}, \ldots, P_{k}^{\lambda_{k}}\right) \\
\quad=\left\{f \in \mathbb{C}\left[x_{0}, x_{1}, x_{2}\right]_{d} \mid f \text { si annulla all'ordine } \lambda_{h} \text { in } P_{h}, h=1, \ldots, k\right\}
\end{aligned}
$$

e supponiamo soddisfatte le 2 condizioni

$$
\begin{aligned}
& \sum_{h=1}^{k} \frac{\lambda_{h}\left(\lambda_{h}-1\right)}{2}=\frac{(d+2)(d+1)}{2}-3, \\
& \sum_{h=1}^{k} \lambda_{h}^{2}=d^{2}-1 .
\end{aligned}
$$

Se supponiamo inoltre che i $P_{1}, \ldots, P_{k}$ sono in posizione generale, allora $\mathcal{C}^{d}\left(P_{1}^{\lambda_{1}}, \ldots, P_{k}^{\lambda_{k}}\right)$ è di dimensione 3 e se per di più la curva generica del sistema è irriducibile, allora ogni base $\varphi=\left(f_{0}, f_{1}, f_{2}\right)$ di questo sistema lineare definisce una trasformazione cremoniana (vedi [4] ibid., §20, p. 158). L'insieme $B(\varphi)=$ $\left\{P_{1}, \ldots, P_{k}\right\}$ si chiama insieme dei punti base, o fondamentali, della trasformazione; essi determinano la trasformazione $\varphi$ a meno di un isomorfismo lineare dello spazio $\mathbb{P}_{\mathbb{C}}^{2}$ di arrivo.

In questo modo però non si ottengono tutte le trasformazioni cremoniane, ma solo quelle cosiddette "generiche", cioè quelle i cui punti base sono punti multipli ordinari; per ottenerle tutte, bisogna ammettere punti base "infinitamente vicini" (vedasi [1] o [4] ibid.).

Se $f_{0}, f_{1}, f_{2} \in \mathbb{R}\left[x_{0}, x_{1}, x_{2}\right]_{d}$, diremo che $\varphi=\left[f_{0}, f_{1}, f_{2}\right]$ è una trasformazione cremoniana reale, e allora $\varphi$ induce una trasformazione $\varphi_{\mathbb{R}}: \mathbb{P}_{\mathbb{R}}^{2} \cdots .>\mathbb{P}_{\mathbb{R}}^{2}$. Nel caso $\mathbb{K}=\mathbb{C}$, si ha sempre che $U(\varphi) \neq \mathbb{P}_{\mathbb{C}}^{2}$, salvo se $d=1$. Se però $f$ è reale, può accadere che $\Sigma(\varphi) \cap \mathbb{P}_{\mathbb{R}}^{2}=\emptyset$, e in tal caso $\varphi_{\mathbb{R}}$ è un diffeomorfismo di $\mathbb{P}_{\mathbb{R}}^{2}$, che noi chiameremo diffeomorfismo birazionale. Notiamo che l'immagine di ogni componente di $\Sigma(\varphi)$ deve essere un punto base dell'inverso di $\varphi$, per cui è equivalente supporre che $\Sigma(\varphi) \cap$ $\mathbb{P}_{\mathbb{R}}^{2}=\emptyset$ o che $B(\varphi) \cap \mathbb{P}_{\mathbb{R}}^{2}=\emptyset$.

È giunto il momento di far vedere l'esempio fondamentale di diffeomorfismo birazionale di $\mathbb{P}_{\mathbb{R}}^{2}$. Siano $P_{1}, P_{2}, P_{3} \in \mathbb{P}_{\mathbb{C}}^{2} \backslash \mathbb{P}_{\mathbb{R}}^{2}$ e siano $\bar{P}_{i}$ i coniugati di $P_{i}, i=1,2,3$; scegliamo $P_{1}, P_{2}, P_{3}$ in modo tale che questi 6 punti non giacciano su una conica; in particolare, $P_{1}, P_{2}$ e $P_{3}$ non devono essere allineati. Per assicurarsi dell'esistenza di tali punti, si può prendere una conica non reale $q$, nel fascio di coniche per $P_{1}, \bar{P}_{1}, P_{2}, \bar{P}_{2}$ poi scegliere $P_{3} \in q \backslash \mathbb{P}_{\mathbb{R}}^{2}$. Il sistema lineare $\mathcal{C}^{5}\left(P_{1}^{2}, P_{2}^{2}, P_{3}^{2}, \bar{P}_{1}^{2}, \bar{P}_{2}^{2}, \bar{P}_{3}^{2}\right)$, cioè il 
sistema delle quintiche aventi un punto doppio in $P_{1}, P_{2}, P_{3}, \bar{P}_{1}, \bar{P}_{2}$ e $\bar{P}_{3}$ soddisfa le condizioni (1) e (2), come si verifica facilmente, e la curva generica è irriducibile; poichè non ha punti base reali, esso definisce un diffeomorfismo di $\mathbb{P}_{\mathbb{R}}^{2}$.

Sia $f_{0}, f_{1}, f_{2}$ una base di $\mathcal{C}^{5}=\mathcal{C}^{5}\left(P_{1}^{2}, P_{2}^{2}, P_{3}^{2}, \bar{P}_{1}^{2}, \bar{P}_{2}^{2}, \bar{P}_{3}^{2}\right)$ e $\varphi=\left[f_{0}, f_{1}, f_{2}\right]$ la trasformazione associata; vediamo ora che il suo inverso è dello stesso tipo. Sia $\gamma_{P_{i}}, i=1,2,3$, la conica per tutti i punti base, eccetto $P_{i}$, e in modo simile $\gamma_{\bar{P}_{i}}$, e scriviamo $\gamma$ per una di queste 6 coniche. Ogni $\gamma$ interseca le quintiche di $\mathcal{C}^{5}$ doppiamente in 5 punti base, e quindi se una $f \in \mathcal{C}^{5}$ passa per un punto generico $P \in \gamma$, allora $f$ contiene $\gamma$ come componente; ne segue che la restrizione di $\mathcal{C}^{5}$ a $\gamma$ è un sistema lineare di dimensione 1, e quindi l'immagine di $\gamma$ si riduce a un punto di $\mathbb{P}^{2}$. Queste coniche appartengono evidentemente alla giacobiana di $\varphi$; questa è di grado 12 , quindi è esattamente la riunione di queste sei coniche. Chiamiamo $P_{i}^{\prime}$ l'immagine di $\gamma_{i}$, e quindi $\bar{P}_{i}^{\prime}$ sarà l'immagine di $\gamma_{\bar{P}_{i}}$; una retta generica interseca una $\gamma$ in due punti, la sua immagine per $\varphi$ sarà dunque una quintica, con punti doppi nei $P_{i}^{\prime}$, e cioè un elemento del sistema lineare $\mathcal{C}^{5}\left(P_{1}^{\prime 2}, \bar{P}_{1}^{\prime 2}, P_{2}^{\prime 2}, \bar{P}_{2}^{\prime 2}, P_{3}^{\prime 2}, \bar{P}_{3}^{\prime 2}\right)$, e quindi se $\psi$ è una trasformazione associata a questo sistema, l'immagine di una retta per la composizione $A=\psi \circ \varphi$ sarà una retta, e allora $A$ è un isomorfismo lineare. Dunque $A^{-1} \circ \psi$ è l'inverso di $\varphi$, il che viene a dire che si può scegliere una base $\psi^{\prime}$ di $\mathcal{C}^{5}\left(P_{1}^{\prime 2}, \bar{P}_{1}^{\prime 2}, P_{2}^{\prime 2}, \bar{P}_{2}^{\prime 2}, P_{3}^{\prime 2}, \bar{P}_{3}^{\prime 2}\right)$ in modo che $\psi^{\prime}$ sia l'inverso di $\varphi$.

Designeremo con $\Phi_{5}$ i sistemi lineari di questo tipo. Si noti che scegliendo punti $P_{1}, P_{2}, P_{3}$ diversi, si ottengono trasformazioni che in generale non sono equivalenti attraverso isomorfismi lineari dello spazio di partenza, visto che la scelta dei $P_{i}$ dipende da 12 parametri reali, mentre il gruppo lineare proiettivo ha solo 8 parametri.

Per semplificare, scriveremo $\mathscr{D}^{d}\left(P_{1}^{\lambda_{1}}, \ldots, P_{k}^{\lambda_{k}}\right)$ per indicare il sistema lineare $\mathcal{C}^{d}\left(P_{1}^{\lambda_{1}}, \bar{P}_{1}^{\lambda_{1}}, \ldots, P_{k}^{\lambda_{k}}, \bar{P}_{k}^{\lambda_{k}}\right)$. Il lettore potrà verificare che i sistemi lineari

$$
\mathscr{D}^{9}\left(P_{1}^{4}, P_{2}^{4}, Q_{1}^{2}, Q_{2}^{2}\right), \quad \mathscr{D}^{13}\left(P^{6}, Q_{1}^{4}, Q_{2}^{4}, Q_{3}^{4}\right) \quad \text { e } \quad D^{13}\left(P_{1}^{6}, P_{2}^{6}, Q_{1}^{2}, Q_{2}^{2}, Q_{3}^{2}\right)
$$

forniscono altri esempi di diffeomorfismi birazionali.

Lo scopo di questo lavoro è di stabilire i due risultati seguenti.

Teorema I. Sia $\varphi: \mathbb{P}_{\mathbb{R}}^{2} \rightarrow \mathbb{P}_{\mathbb{R}}^{2}$ un diffeomorfismo birazionale di ordine d. Allora $d \equiv 1 \bmod 4$.

Teorema II. Ogni diffeomorfismo birazionale di $\mathbb{P}_{\mathbb{R}}^{2} \grave{e}$ composizione di diffeomorfismi di tipo $\Phi_{5}$.

La prova del teorema I è elementare e sarà presentata alla fine di questa introduzione.

Il teorema II può essere considerato come una versione reale del noto teorema di Noether, che afferma che ogni trasformazione birazionale di $\mathbb{P}_{\mathbb{C}}^{2}$ si può scrivere 
come composizione di trasformazioni quadratiche con 3 punti base distinti. La sua dimostrazione è più elaborata; quella che proponiamo è ispirata dal programma di Sarkisov (vedere [9] § 1.8, [3]). L'idea di base è quella già sfruttata da Noether: nel caso complesso, per una trasformazione di ordine $d$, si mostra che le 3 maggiori molteplicità $\lambda_{1}, \lambda_{2}, \lambda_{3}$ dei punti base soddisfano l'ineguaglianza

$$
\lambda_{1}+\lambda_{2}+\lambda_{3}>d
$$

Questi 3 punti vengono poi utilizzati, quando è possibile, come punti base di una trasformazione quadratica per semplificare la trasformazione iniziale (cioè, abbassarne l'ordine).

La versione reale di questo risultato afferma che, per un diffeomorfismo birazionale di $\mathbb{P}_{\mathbb{R}}^{2}$, esistono 3 paia di punti base, $P_{1}, P_{2}, P_{3}$ e i loro coniugati $\bar{P}_{1}, \bar{P}_{2}, \bar{P}_{3}$, che pure soddisfano l'ineguaglianza qui sopra. Questi 6 punti possono essere utilizzati come punti base di una trasformazione di tipo $\Phi_{5}$ per semplificare la trasformazione iniziale. La difficoltà, nel nostro caso come per il teorema di Noether, consiste nel fatto che i punti base di maggiore molteplicità non sempre sono dei bravi punti distinti di $\mathbb{P}_{\mathbb{R}}^{2}$, ma alcuni possono essere "infinitamente prossimi". Per superare questa difficoltà è utile, seguendo appunto l'idea di Sarkisov, di aver ricorso a modificazioni appropriate dello spazio $\mathbb{P}_{\mathbb{R}}^{2}$, e cioè le superfici $Q$ e $T$ (fibrati di Mori) di cui parleremo al 3 .

Non esistono esempi simili nel caso della retta proiettiva, poiché le trasformazioni cremoniane in questo caso sono isomorfismi lineari. Esistono pertanto diffeomorfismi $\varphi: \mathbb{P}_{\mathbb{R}}^{1} \rightarrow \mathbb{P}_{\mathbb{R}}^{1}$ della forma $\varphi=\left[f_{0}, f_{1}\right]$, con $f_{0}, f_{1} \in \mathbb{R}\left[x_{0}, x_{1}\right]$ di grado superiore a 1. Ad esempio:

$$
f_{0}(t)=\frac{t^{3}+t}{t^{2}+2}
$$

Si verifica che $f_{0}^{\prime}(t) \neq 0$ per tutti $t \in \mathbb{R}$; d'altra parte, se si pone

$$
f_{1}(u)=1 / f_{0}(1 / u)=\frac{2 u^{3}+u}{u^{2}+1}
$$

di nuovo si ha che $f_{1}^{\prime}(u) \neq 0$ per tutti $u \in \mathbb{R}$. Quindi $\varphi$ è un diffeomorfismo razionale di $\mathbb{P}_{\mathbb{R}}^{1}$, il cui inverso però non può essere razionale. Questo esempio si può visualizzare così: si consideri la curva parametrica $\alpha: t \mapsto\left(t^{2}+1, t^{3}+t\right)$; è ovviamente una cubica razionale, che possiede un punto doppio isolato sui reali in $(0,0)$, immagine di $t= \pm \sqrt{-1}$. La composizione di $\alpha$ con la proiezione dal punto doppio $(0,0)$ è l'identità. Se proiettiamo invece da un punto vicino $P$ a $(0,0)$, sui reali, si ottiene una mappa prossima all'identità, quindi un diffeomorfismo (mentre sui complessi si avrà una mappa di grado 3$)$. Scegliendo $P=(-1,0)$ si ottiene appunto la mappa $f_{0}(t)$ qui sopra. 
Per concludere questa introduzione, vediamo un esempio di diffeomorfismo birazionale di $\mathbb{P}_{\mathbb{R}}^{3}$. Si prendano 6 punti $P_{1}, P_{2}, P_{3}$ e $\bar{P}_{1}, \bar{P}_{2}, \bar{P}_{3}$ in $\mathbb{P}_{\mathbb{C}}^{2}$ come per una trasformazione di tipo $\Phi_{5}$. Si scelga una base $\alpha=\left(\alpha_{0}, \ldots, \alpha_{3}\right)$ dello spazio vettoriale di dimensione 4 delle cubiche piane che passano per i $P_{i}$ ed i $\bar{P}_{i}, i=1,2,3$, cosicchè $\alpha\left(\mathbb{P}_{\mathbb{C}}^{2}\right) \subset \mathbb{P}_{\mathbb{C}}^{3}$ è una superfice cubica reale. Si prenda una quartica liscia, reale $\Gamma_{4} \subset \mathbb{P}_{\mathbb{C}}^{2}$ senza punti reali, che passa per i $P_{i}$ ed i $\bar{P}_{i}, i=1,2,3$ (se avete dubbi sull'esistenza di una tale quartica, prendete prima la quartica $x_{0}^{4}+x_{1}^{4}+x_{2}^{4}=0$, e poi $P_{1}, P_{2}, P_{3}$ su questa quartica). Allora $\Gamma_{6}=\alpha\left(\Gamma_{4}\right)$ è una sestica reale, senza punti reali. Com'è noto (vedi [10], chap. VII, §4.2, o [7]), il sistema lineare delle superfici cubiche che contengono la $\Gamma_{6}$ fornisce una trasformazione birazionale di $\mathbb{P}_{\mathbb{C}}^{3}$, con $\Gamma_{6}$ come curva di punti base; prendendo una base reale di questo sistema lineare si ottiene quindi un diffeomorfismo birazionale di $\mathbb{P}_{\mathbb{R}}^{3}$.

Convenzione. Seguendo [8], una varietà reale è una varietà proiettiva $X$ sul corpo dei complessi, munita di una struttura reale, cioè di un anti-automorfismo involutivo $x \mapsto \bar{x}$; si denota con $X(\mathbb{R})$ l'insieme dei punti reali, cioè tali che $\bar{x}=x$. Per esempio, lo spazio proiettivo complesso $\mathbb{P}_{\mathbb{C}}^{n}$, munito della coniugazione delle coordinate omogenee: $\left[z_{0}: \cdots: z_{n}\right] \mapsto\left[\bar{z}_{0}: \cdots: \bar{z}_{n}\right]$; d'ora in poi denoteremo con $\mathbb{P}^{n}$ questo spazio, e quindi abbiamo $\mathbb{P}^{n}(\mathbb{R})=\mathbb{P}_{\mathbb{R}}^{n}$. Un altro esempio tipico è una varietà proiettiva complessa definita da equazioni polinomiali a coefficienti reali, come la quadrica $Q \subset \mathbb{P}^{3}$ definita da $z_{0}^{2}+z_{1}^{2}+z_{2}^{2}-z_{3}^{2}=0$, per la quale $Q(\mathbb{R})$ è la sfera.

Utilizzeremo il gruppo $\mathcal{N}_{1}(X)$ di Néron-Severi, che è il quoziente dello spazio vettoriale $($ su $\mathbb{R})$ generato dai cicli reali di dimensione 1 su $X$ per la relazione dell'equivalenza numerica: un ciclo $C \subset X$ di dimensione 1 è reale se $\bar{C}=C$ e due cicli $C$ e $C^{\prime}$ sono equivalenti se $(C \cdot D)=\left(C^{\prime} \cdot D\right)$ per ogni divisore di Cartier $D$ su di $X$. Scriveremo $|D|$ per il sistema lineare completo costituito dai divisori complessi linearmente equivalenti a $D$. Salvo menzione esplicita, le varietà e morfismi considerati saranno reali.

Prova del teorema I. Sia dunque $\varphi: \mathbb{P}^{2} \ldots . .>\mathbb{P}^{2}$ un diffeomorfismo birazionale di ordine $d$. La giacobiana $\Sigma(\varphi)$ è una curva di grado $3(d-1)$; poiché non ha punti reali, le sue componenti devono essere di grado pari. Non può avere componenti invarianti per la coniugazione complessa, poiché la sua immagine sarebbe un punto base reale dell'inverso. Quindi $\Sigma(\varphi)$, essendo composta da un numero pari di componenti, ciascuna di grado pari, il suo grado è un multiplo di 4 :

$$
3(d-1) \equiv 0 \bmod 4 \Rightarrow d \equiv 1 \bmod 4 .
$$




\section{L'ineguaglianza di Noether}

Come è spiegato in [1], specialmente nella proposizione 2.5.2 (o anche in [4] ibid., o [10] ibid.), una trasformazione cremoniana può sempre essere descritta prendendo una base del sistema lineare $\mathcal{C}\left(P_{1}^{\lambda_{1}}, \ldots, P_{k}^{\lambda_{k}}\right)$ delle curve di grado $d$ che passano per dei punti $P_{1}, \ldots, P_{k}$, con molteplicità $\lambda_{1}, \ldots, \lambda_{k}$. Alcuni di questi punti però possono essere "infinitamente vicini". Comunque, le seguenti formule restano valide:

$$
\begin{aligned}
& \frac{(d-1)(d-2)}{2}-\sum_{i=1}^{k} \frac{\lambda_{i}\left(\lambda_{i}-1\right)}{2}=0, \\
& \sum_{i=1}^{k} \lambda_{i}^{2}=d^{2}-1 .
\end{aligned}
$$

La (1) esprime che la curva deve essere di genero uguale a 0 , la (2) che due curve del sistema si intersecano, oltre ai punti basi, in un solo punto variabile. Se ne deducono le due uguaglianze equivalenti:

$$
\begin{aligned}
& \sum_{i=1}^{k} \lambda_{i}=3(d-1), \\
& \sum_{i=1}^{k} \lambda_{i}^{2}=d^{2}-1 .
\end{aligned}
$$

Queste uguaglianze possono essere sfruttate per dimostrare che se $P_{1}, P_{2}$ e $P_{3}$ sono i punti base con massima molteplicità di un morfismo birazionale che non sia un isomorfismo(cioè $d>1$ ), si ha l'ineguaglianza di Noether:

$$
\lambda_{1}+\lambda_{2}+\lambda_{3}>d
$$

come ad esempio in [4], libro quinto, capitolo II, p. 166. Osserviamo che i punti base sono almeno 3 , perché se no $\lambda_{1}+\lambda_{2}=3(d-1)$ e allora la retta per $P_{1}$ e $P_{2}$ si staccherebbe dalle curve del sistema.

Nel caso di un diffeomorfismo birazionale, i punti basi, anche quelli infinitamente vicini, vengono per paia di punti coniugati $P_{i} \neq \bar{P}_{i}, i=1, \ldots, k$ con stessa molteplicità $\lambda_{i}$. In questo caso sappiamo inoltre che il grado del sistema è della forma $d=4 m+1$, che scriviamo $\mathscr{D}^{d}\left(P_{1}^{\lambda_{1}}, \ldots, P_{k}^{\lambda_{k}}\right)$. Dimostriamo ora il lemma seguente, che è la versione adeguata dell'ineguaglianza di Noether. Sottolineamo che nell'enunciato, le tre paia $\left(P_{1}, \bar{P}_{1}\right),\left(P_{2}, \bar{P}_{2}\right)$ e $\left(P_{3}, \bar{P}_{3}\right)$ sono distinte.

Osservazione. Se $\lambda_{1} \geq \lambda_{2} \geq \lambda_{3}$, l'ineguaglianza di Noether classica ci dice solo che $2 \lambda_{1}+\lambda_{2} \geq 4 m+1$. 
Lemma (ineguaglianza di Noether per un diffeomorfismo birazionale). Siano $P_{1}, P_{2}$ e $P_{3}$ tre punti base con la massima molteplicità. Allora si ha l'ineguaglianza

$$
\lambda_{1}+\lambda_{2}+\lambda_{3}>4 m+1 .
$$

Prova. Seguiamo la prova di [6], chap. I, § 10. Possiamo supporre che $\lambda_{1} \geq \lambda_{2} \geq \lambda_{3}$. Segue da $\left(1^{\prime}\right)$ e (2) che

$$
\begin{aligned}
& \sum_{i=1}^{k} \lambda_{i}=\frac{1}{2} 3(4 m+1-1)=6 m, \\
& \sum_{i=1}^{k} \lambda_{i}^{2}=\frac{1}{2}\left((4 m+1)^{2}-1\right)=4 m(2 m+1) .
\end{aligned}
$$

Notiamo che $\lambda_{1} \leq 2 m$, se no la retta per $P_{1}$ e $\bar{P}_{1}$ si staccherebbe come componente fissa.

Calcoliamo (b) $-\lambda_{3} \cdot(a)$ :

$$
\sum_{i=1}^{k} \lambda_{i}^{2}-\lambda_{3} \cdot \sum_{i=1}^{k} \lambda_{i}=2 m(4 m+2)-6 m \cdot \lambda_{3}
$$

che si può riscrivere

$$
\lambda_{1}\left(\lambda_{1}-\lambda_{3}\right)+\lambda_{2}\left(\lambda_{1}-\lambda_{3}\right)-\sum_{i=4}^{k} \lambda_{i}\left(\lambda_{3}-\lambda_{i}\right)=2 m(4 m+2)-6 m \lambda_{3}
$$

o ancora

$$
2 m(4 m+2)=\lambda_{1}\left(\lambda_{1}-\lambda_{3}\right)+\lambda_{2}\left(\lambda_{1}-\lambda_{3}\right)-\sum_{i=4}^{k} \lambda_{i}\left(\lambda_{3}-\lambda_{i}\right)+6 m \lambda_{3} .
$$

Ne deduciamo

$$
\begin{aligned}
& 2 m\left(\lambda_{1}+\lambda_{2}+\lambda_{3}-(4 m+2)\right) \\
& \quad=2 m\left(\lambda_{1}+\lambda_{2}+\lambda_{3}\right)-\lambda_{1}\left(\lambda_{1}-\lambda_{3}\right)-\lambda_{2}\left(\lambda_{1}-\lambda_{3}\right)+\sum_{i=4}^{k} \lambda_{i}\left(\lambda_{3}-\lambda_{i}\right)-6 m \lambda_{3} \\
& \quad=\left(2 m-\lambda_{1}\right)\left(\lambda_{1}-\lambda_{3}\right)+\left(2 m-\lambda_{2}\right)\left(\lambda_{2}-\lambda_{3}\right)+\sum_{i=4}^{k} \lambda_{i}\left(\lambda_{3}-\lambda_{i}\right) .
\end{aligned}
$$

Poiché tutti i termini dell'ultima espressione sono positivi o zero, se ne deduce che $\lambda_{1}+\lambda_{2}+\lambda_{3}-(4 m+2) \geq 0$ e quindi $\lambda_{1}+\lambda_{2}+\lambda_{3}>4 m+1$. 
Per incoraggiarci, notiamo che se $\psi$ è un diffeomorfimo birazionale di grado $4 m+1$ i cui tre punti di massima molteplicità $P_{1}, P_{2}, P_{3}$ sono dei veri punti di $\mathbb{P}^{2}$, questi e i loro coniugati non possono essere su una stessa conica $q$, perché se no segue dall'ineguaglianza di Noether che $q$ interseca una curva del sistema di $\psi$ in almeno $2\left(\lambda_{1}+\lambda_{2}+\lambda_{3}\right)>2 \cdot(4 m+1)$ punti, e quindi si staccherebbe dal sistema di $\psi$. Possiamo dunque scegliere una trasformazione $\varphi$ di tipo $\Phi_{5}$ con punti base i $P_{i}$ e i loro coniugati; una curva del sistema di $\varphi$ e una del sistema di $\psi$ si incontrano nei punti base, che contano per $2\left(2\left(\lambda_{1}+\lambda_{2}+\lambda_{3}\right)\right) \geq 4(4 m+2)$ punti, ed inoltre in un numero di punti liberi uguale all'ordine di $\psi \circ \varphi^{-1}$, che quindi è uguale a

$$
5 \cdot(4 m+1)-4\left(\lambda_{1}+\lambda_{2}+\lambda_{3}\right)=4 m+1-4\left(\lambda_{1}+\lambda_{2}+\lambda_{3}-(4 m+1)\right)
$$

ed è perciò strettamente inferiore all'ordine di $\psi$.

\section{Alcuni modelli di $\mathbb{P}^{2}$}

Lo scopo di questo paragrafo è di definire le superfici che utilizzeremo per semplificare un diffeomorfismo birazionale, e di stabilirne alcune proprietà.

Sia $Q$ la quadrica reale di $\mathbb{P}^{3}$ definita dall' equazione $z_{0}^{2}+z_{1}^{2}+z_{2}^{2}-z_{3}^{2}=0$ e sia $T$ la superfice ottenuta facendo scoppiare due punti immaginari coniugati $A, \bar{A}$ su $Q$; si verifica facilmente che il gruppo di Lorentz (cioè il gruppo che lascia invariante l'equazione di Q) opera transitivamente su $Q \backslash Q(\mathbb{R})$, e quindi diverse scelte dei due punti danno luogo a varietà isomorfe. Notiamo che $Q$ è isomorfo a $\mathbb{P}^{1} \times \mathbb{P}^{1}$, per il morfismo

$$
\left.\mathbb{P}^{1} \times \mathbb{P}^{1} \rightarrow \mathbb{P}^{3}, \quad([a, b],[u, v]) \mapsto[i(a u+b v), a u-b v, a v+b u, a v-b u)\right] ;
$$

il quale è compatibile con la coniugazione su $\mathbb{P}^{1} \times \mathbb{P}^{1}$ definita da $[(a, b),(u, v)] \rightarrow$ $[(\bar{v},-\bar{u}),(\bar{b},-\bar{a})]$, e la coniugazione usuale su $\mathbb{P}^{3}$.

Lavoreremo con la categoria $\&$ (in omaggio a Sarkisov) i cui oggetti sono le superfici reali $\mathbb{P}^{2}, Q$ e $T$, con la loro struttura reale, e i cui morfismi sono le trasformazioni birazionali. In più, per ogni oggetto $S$ di $\&$ si distingue un elemento $F_{S}$ particolare di $\mathcal{N}_{1}(S)$ :

- $F_{\mathbb{P}^{2}}$ è la classe di una retta reale.

- $F_{Q}$ è la classe di una curva di tipo $(1,1)$. Poichè la coniugazione di $Q$ manda le curve complesse di tipo $(m, n)$ su quelle di tipo $(n, m), F_{Q}$ è un generatore di $\mathcal{N}_{1}(Q)$.

- $F_{T}=\pi^{*}\left(F_{Q}\right)-E_{\pi}$, dove $\pi: T \rightarrow Q$ denota lo scoppiamento dei due punti immaginari coniugati $A, \bar{A}$ e $E_{\pi}$ la classe del divisore eccezionale, cioè $E_{\pi}=$ $\pi^{-1}(\{A, \bar{A}\})$. 
Secondo l'usanza, designeremo con $K_{S}$ il divisore canonico della superfice $S$. Notiamo che $K_{\mathbb{P}^{2}}=-3 F_{\mathbb{P}^{2}}$ e che $F_{\mathbb{P}^{2}}^{2}=1$. D'altra parte, $K_{Q}=-2 \cdot F_{Q}$ e quindi possiamo scrivere $K_{Q}=-2(1,1)$ e $F_{Q}^{2}=(1,1) \cdot(1,1)=2$.

Passiamo a $T$, che è un caso più elaborato; si vedrà che $T$ è un fibrato in coniche su $\mathbb{P}^{1}$. Ricordiamo che un fibrato in coniche è un morfismo $f: S \rightarrow B$ di una superfice $S$ su una curva $B$, tale che la fibra generica è isomorfa a $\mathbb{P}^{1}$, e le altre (in numero finito) sono isomorfe all'unione di due rette che si intersecano trasversalmente in un punto (vedi [2]).

Proposizione (proprietà e caratterizzatione di $T$ ).

i) $\mathcal{N}_{1}(T)$ è di dimensione 2 , ed è generato da $K_{T}$ e $F_{T}$; per l'intersezione, si ha

$$
F_{T}^{2}=0, \quad K_{T} \cdot F_{T}=-2, \quad K_{T}^{2}=6 .
$$

ii) Il sistema lineare $\left|F_{T}\right|$ è di dimensione 1 , senza punti base; il morfismo $\varphi: T \rightarrow$ $\mathbb{P}^{1}$ che se ne deduce è un fibrato in coniche con esattamente due fibre singolari, che sono situate su due punti reali di $\mathbb{P}^{1}$, e sono costituite ciascuna da due rette immaginarie coniugate.

iii) Reciprocamente, sia $\gamma: X \rightarrow \mathbb{P}^{1}$ un fibrato in coniche con esattamente due fibre singolari, situate su due punti reali di $\mathbb{P}^{1}$; supponiamo che $\mathcal{N}_{1}(X)$ sia di dimensione 2. Allora esiste un morfismo $\pi_{X}: X \rightarrow Q$ che presenta $X$ come lo scoppiamento di due punti immaginari coniugati di $Q$.

Prova. i) Sia $\pi: T \rightarrow Q$ lo scoppiamento di due punti $A, \bar{A} \in Q$ e sia $E_{\pi}=$ $\pi^{-1}(\{A, \bar{A}\})$. Allora è chiaro che $\mathcal{N}_{1}(T)$ è generato da $\pi^{*}\left(F_{Q}\right)$ e $E_{\pi}$. Poichè $K_{T}=$ $\pi^{*}\left(K_{Q}\right)+E_{\pi}$, ne seguono le formule e asserzioni di i).

ii) Consideriamo la proiezione $\mathbb{P}^{3} \quad \cdots .>\mathbb{P}^{1}$ centrata sulla retta che passa per $A$ e $\bar{A}$, e la sua restrizione $\varphi^{\prime}: Q \quad>\mathbb{P}^{1}$. Le fibre di $\varphi^{\prime}$ sono le intersezioni dei piani per $A$, $\bar{A}$ con $Q$, e quindi sono di tipo $(1,1)$; inoltre, esattamente due di queste degenerano in un paio di rette immaginarie, e cioè quelle che corrispondono ai piani tangenti a $Q$ che passano per $A$ e $\bar{A}$. L'asserzione ii) segue poi dal fatto che $\varphi=\varphi^{\prime} \circ \pi$.

iii) Qui è meglio dividere la prova in più tappe.

a) $\mathcal{N}_{1}(X)$ è generato da $K_{X}$ e dalla fibra $F_{X}$ di $\gamma$; si ha

$$
F_{X}^{2}=0, \quad F_{X} \cdot K_{X}=-2, \quad K_{X}^{2}=6 .
$$

È chiaro che $F_{X}^{2}=0$ e segue dalla formula dell' aggiunta che $F_{X} \cdot K_{X}=-2$; quindi $F_{X}$ e $K_{X}$ sono una base di $\mathcal{N}_{1}(X)$.

Se ci fosse una fibra singolare $F_{1}=C^{\prime} \cup C^{\prime \prime}$, con componenti $C^{\prime}$ e $C^{\prime \prime}$ reali, allora si avrebbe:

$$
0=\left(C^{\prime}+C^{\prime \prime}\right)^{2}=\left(C^{\prime}\right)^{2}+2 \underbrace{C^{\prime} \cdot C^{\prime \prime}}_{=1}+\left(C^{\prime \prime}\right)^{2} \Rightarrow\left(C^{\prime}\right)^{2}+\left(C^{\prime \prime}\right)^{2}=-2
$$


quindi o $\left(C^{\prime}\right)^{2} \neq 0$, o $\left(C^{\prime \prime}\right)^{2} \neq 0$; mettiamo che $\left(C^{\prime}\right)^{2} \neq 0$. Si può scrivere $C^{\prime}=a \cdot F_{X}+b \cdot K_{X}, a, b \in \mathbb{R}$. Allora

$$
0=F_{X} \cdot C^{\prime}=a \cdot 0-2 \cdot b \Rightarrow b=0 .
$$

Ma allora $\left(C^{\prime}\right)^{2}=\left(a \cdot F_{X}\right)^{2}=0$, una contraddizione.

Quindi, le 2 fibre singolari $F_{1}$ e $F_{2}$ sono ciascuna riunione di due curve immaginarie coniugate: $F_{i}=C_{i} \cup \bar{C}_{i}$ che si intersecano trasversalmente in un punto.

Lavorando per un momento con cicli complessi su $X(\mathbb{C})$ e le loro classi di equivalenza numerica, vediamo ora che $C_{i}^{2}=\bar{C}_{i}^{2}=-1, i=1,2$. Per prima cosa, osserviamo che se $C, D \subset X$ sono due cicli complessi, allora $C \cdot D=\bar{C} \cdot \bar{D}$, perché la coniugazione preserva l'orientazione della superfice e rovescia quella dei cicli. Scrivendo $C$ per $C_{1}$ o $C_{2}$ :

$$
0=F_{X}^{2}=(C+\bar{C})^{2}=C^{2}+2 \underbrace{C \cdot \bar{C}}_{=1}+\bar{C}^{2} \Rightarrow C^{2}=\bar{C}^{2}=-1 .
$$

Possiamo quindi considerare il morfismo $\sigma: X \rightarrow \mathbb{F}$ che contrae $C_{1}$ e $C_{2}$ : la superfice $\mathbb{F}$ non è reale, e $\sigma$ è un morfismo di superfici complesse. La $\mathbb{F}$ è una superfice rigata, e si ha un diagramma commutativo:

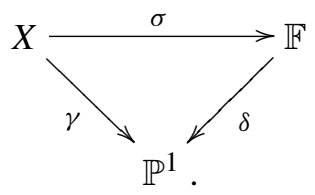

È risaputo che $K_{\mathbb{F}}^{2}=8$ (vedi [5], chap. V, corollary 2.11), e ne segue che $K_{X}^{2}=6$.

b) Non esistono sezioni definite sui reali di $\gamma: X \rightarrow \mathbb{P}^{1}$. Se una tale sezione esistesse, notiamo con $C$ la classe della sua immagine in $\mathcal{N}_{1}(X)$; poichè

$$
C \cdot F_{X}=1 \quad \text { e } \quad C^{2}+C \cdot K_{X}=-2 \quad \text { (formula dell'aggiunta) }
$$

e ponendo $C=\alpha K_{X}+\beta F_{X}, \alpha, \beta \in \mathbb{R}$, si avrebbe

$$
-2 \alpha=1,6 \alpha(\alpha+1)-2 \beta(1+2 \alpha)=-2 \Rightarrow 6(-1 / 2)(1 / 2)=-2
$$

il ché è assurdo.

c) $X$ è lo scoppiamento di due punti su $Q$. La rigata complessa $\mathbb{F}$ è della forma $\mathbb{P}(\mathcal{O}(0) \oplus \mathcal{O}(-k))$, con $k \geq 0$. Ora vediamo quali valori può avere $k$. Osserviamo che $\sigma: X \rightarrow \mathbb{F}$ induce una biiezione fra le sezioni di $\gamma$ e quelle di $\delta$ : alla sezione $D \subset X$ di $\gamma$ si associa $\sigma(D)$, e alla $D^{\prime} \subset \mathbb{F}$ di $\delta$ si associa il trasformato stretto $\sigma^{-1}\left(D^{\prime}\right)$. Siano infine $P_{i}=\sigma\left(C_{i}\right), i=1,2$, i due punti che $\sigma$ fa scoppiare. 
Il caso $k=0$. Allora $\mathbb{F}=\mathbb{P}^{1} \times \mathbb{P}^{1}$, e si può supporre che $\delta$ è la proiezione sul primo fattore; le sezioni complesse di $\delta$ sono le curve irriducibili $D_{1, n}$ di tipo $(1, n)$, con $n \geq 0$. Ne segue

$$
\left(\sigma^{-1}\left(D_{1, n}\right)\right)^{2}= \begin{cases}2 n & \text { se } P_{i} \notin D_{1, n}, i=1,2, \\ 2 n-2 & \text { se } P_{i} \in D_{1, n}, i=1,2, \\ 2 n-1 & \text { negli altri casi. }\end{cases}
$$

Quindi si ha una delle seguenti possibilità; fra tutte le sezioni di $\gamma$ :

(i) Esiste esattamente una $D_{0}$ con $D_{0}^{2}<0$, e cioè $D_{0}=\sigma^{-1}\left(D_{1,0}\right), D_{1,0} \ni P_{1}, P_{2}$.

(ii) Esistono esattamente due sezioni $D_{1}$ e $D_{2}$ con $D_{1}^{2}, D_{2}^{2}<0$, trasformate strette di $D_{1,0}^{(1)} \ni P_{1}$ e $D_{1,0}^{(2)} \ni P_{2}$ rispettivamente. Per di più, $D_{i}^{2}=-1$ e $D_{1} \cap D_{2}=\emptyset$.

Il caso (i) non può presentarsi, poiché $D_{0}$ sarebbe una sezione reale, in contraddizione con $b$ ).

Nel caso (ii), si ha $D_{2}=\bar{D}_{1}$, cosicché la contrazione $\pi_{X}: X \rightarrow X^{\prime}$ di $D_{1}+$ $\bar{D}_{1}$ è reale. Infine, $X(\mathbb{R})$ è omeomorfa alla sfera, quindi anche $X^{\prime}(\mathbb{R})$, e poiché $\operatorname{dim}\left(\mathcal{N}_{1}\left(X^{\prime}\right)\right)=\operatorname{dim}\left(\mathcal{N}_{1}(X)\right)-1=1$, segue dal lemma 1.16 di [8] che $X^{\prime}$ è isomorfo a $Q$.

Il caso $k>0$. In questo caso esiste un'unica sezione $D_{0}$ di $\delta$ di self-intersezione negativa: $D_{0}^{2}=-k$; tutte le altre sezioni sono della forma $D_{0}+n \cdot F$ con $n \geq k$, dove $F$ è la fibra di $\delta$, e quindi

$$
\left(D_{0}+n \cdot F\right)^{2}=-k+2 n \geq n \geq k>0
$$

(vedi [5], chap, V, theorem 2.17).

Il caso $k \geq 2$ è escluso, perché allora $\sigma^{-1}\left(D_{0}\right)$ sarebbe l'unica sezione di $\gamma$ con self-intersezione negativa, quindi reale, in contraddizione con $b$ ).

Se $k=1$, le uniche sezioni complesse di $\gamma$ con self-intersezione negativa sono:

(i) $\sigma^{-1}\left(D_{0}+F\right)$ quando $D_{0}+F$ contiene $P_{1}$ e $P_{2}$, e allora $\sigma^{-1}\left(D_{0}+F\right)^{2}=-1$.

(ii) $\sigma^{-1}\left(D_{0}\right)$, e allora $\sigma^{-1}\left(D_{0}\right)^{2}=-1-\left|D_{0} \cap\left\{P_{1}, P_{2}\right\}\right|$. Se $D_{0}$ contiene $P_{1}$ o $P_{2}$, allora $\sigma^{-1}\left(D_{0}\right)$ è l'unica sezione di $\gamma$ con self-intersezione $<-1$, e quindi sarebbe reale, in contraddizione con b). Quindi $P_{1}, P_{2} \notin D_{0}$ e $\sigma^{-1}\left(D_{0}\right)$ ha selfintersezione -1 . Sia $\varphi: \mathbb{F} \rightarrow \mathbb{P}^{2}$ la contrazione di $D_{0}$. Allora $F=\varphi^{*} H-D_{0}$, ove $H$ è una retta di $\mathbb{P}^{2}$; le sezioni complesse di $\delta$ del tipo $D_{0}+F$ corrispondono per $\varphi$ alle rette di $\mathbb{P}^{2}$ che non passano per $\varphi\left(D_{0}\right)$. Esiste quindi esattamente una sezione $D_{P_{1}, P_{2}}$ del tipo $D_{0}+F$ che passa per $P_{1}$ e $P_{2}$ e ne segue che le uniche sezioni di $\gamma$ con self-intersezione negativa, in realtà uguale $\mathrm{a}-1$, sono $\sigma^{-1}\left(D_{0}\right)$ e $\sigma^{-1}\left(D_{P_{1}, P_{2}}\right)$. Proseguiamo ora come nel (ii) del caso $k=0$ : abbiamo che $\overline{\sigma^{-1}\left(D_{0}\right)}=\sigma^{-1}\left(D_{P_{1}, P_{2}}\right)$, l'intersezione di $\sigma^{-1}\left(D_{0}\right)$ e $\sigma^{-1}\left(D_{P_{1}, P_{2}}\right)$ è vuota, e la contrazione di queste due sezioni presenta $X$ come scoppiamento di un paio di punti immaginari coniugati di $Q$. 
Una prova succinta di iii) si trova in [8], lemma 3.2.4. Osserviamo che i due casi della parte c) della prova di iii) qui sopra si presentano effettivamente. Difatti, partendo da $\pi: T \rightarrow Q$, chiamiamo $E_{\pi}^{\prime}$ e $E_{\pi}^{\prime \prime}$ le componenti della fibra eccezionale di $\pi, C^{\prime} \cup \bar{C}^{\prime}$ e $C^{\prime \prime} \cup \bar{C}^{\prime \prime}$ le due fibre singolari, dove $C^{\prime} \cap E_{\pi}^{\prime} \neq \emptyset$ e $C^{\prime \prime} \cap E_{\pi}^{\prime \prime} \neq \emptyset$. Se definiamo $\mathbb{F}$ contraendo $C^{\prime}$ e $C^{\prime \prime}$, allora $\mathbb{F} \simeq \mathbb{P}(\mathcal{O}(0) \oplus \mathcal{O}(-1))$; se prendiamo invece $C^{\prime}$ e $\bar{C}^{\prime \prime}$, allora $\mathbb{F} \simeq \mathbb{P}^{1} \times \mathbb{P}^{1}$.

\section{Prova del teorema II}

In generale, per una trasformazione birazionale $\varphi=\left[f_{0}, f_{1}, f_{2}\right]: S \cdots>\mathbb{P}^{2}$, designeremo con $\mathscr{H}_{\varphi}$ il sistema lineare generato dalle $f_{i}$ e con $H_{\varphi}$ la classe di un rappresentante di $\mathscr{H}_{\varphi}$ in $\mathcal{N}_{1}(S)$.

Il sistema lineare $\mathcal{H}_{\varphi}$ determina la trasformazione $\varphi$ a meno di un isomorfismo lineare sul $\mathbb{P}^{2}$ di arrivo; quindi, nel caso di $\varphi: \mathbb{P}^{2} \quad \ldots \mathbb{P}^{2}$, l'ordine $d$ di $\varphi$ è determinato da $H_{\varphi} \in \mathcal{N}_{1}\left(\mathbb{P}^{2}\right)$, perché $H_{\varphi}=d \cdot F_{\mathbb{P}^{2}}$.

4.1. Semplificazione alla Sarkisov. Sia $\psi: \mathbb{P}^{2} \ldots .>\mathbb{P}^{2}$ un diffeomorfismo birazionale che non è un isomorfismo. Descriveremo una sequenza di modifiche:

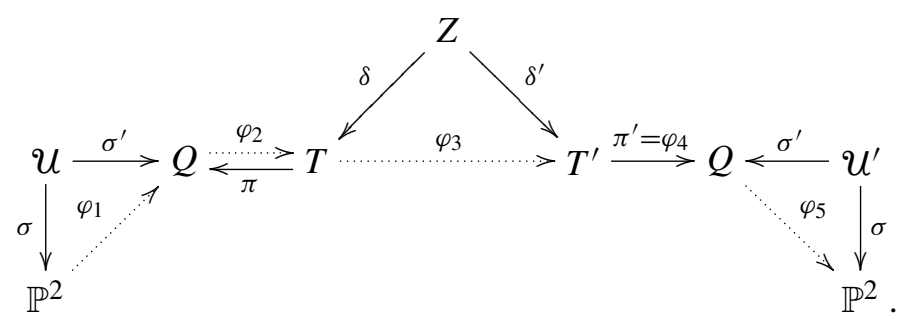

Chiameremo $\psi_{h}$ la composizione di $\psi=\psi_{0}$ con $\left(\varphi_{h} \circ \cdots \circ \varphi_{1}\right)^{-1}, h=1, \ldots, 5$, e scriveremo $H_{h}$ per $H_{\psi_{h}}$ e $\mathscr{H}_{h}$ per $\mathscr{H}_{\psi_{h}}$ :

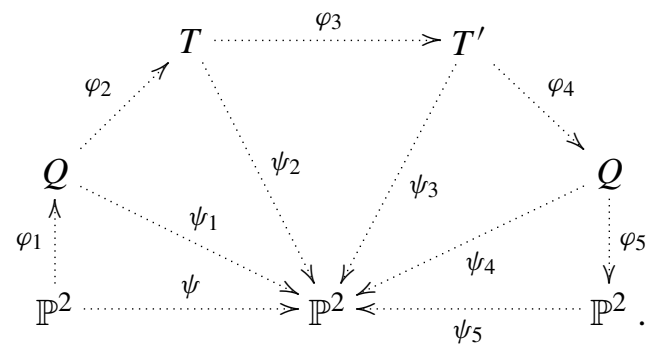

Vedremo che $H_{5}=d^{\prime} \cdot F_{\mathbb{P}^{2}}$, con $d^{\prime} \leq d-4$ e che $\varphi_{5} \circ \cdots \circ \varphi_{1}$ è un diffeomorfismo birazionale di ordine 5 , non necessariamente di tipo $\Phi_{5}$, ma eventualmente una 
degenerazione di queste, in cui due o tre paia di punti base vengono a coincidere; per dimostrare il teorema II, occorrerà esprimere quest'ultime trasformazioni come composizione di trasformazioni di tipo $\Phi_{5}$, il ché faremo al $\S 4.2$.

In generale, se $\pi: X \rightarrow Y$ è lo scoppiamento di uno o più punti di $Y$, denoteremo con $E_{\pi}$ il divisore eccezionale di $\pi$.

Prima mossa. Sia $A \in \mathbb{P}^{2}$ un punto base di $\mathscr{H}_{\psi}$ di massima molteplicità $\lambda_{1}$. Definiamo $\varphi_{1}$ con il triangolo birazionale

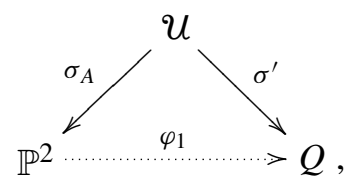

dove $\sigma=\sigma_{A}$ è lo scoppiamento dei due punti $A$ e $\bar{A}$, e $\sigma^{\prime}$ la contrazione della retta reale per $A$ e $\bar{A}$. Si ha

$$
H_{1}=\sigma_{*}^{\prime}\left(\sigma^{*}\left(H_{0}\right)-\lambda_{1} \cdot E_{\sigma}\right)=\sigma_{*}^{\prime}\left(d \cdot\left(E_{\sigma}+E_{\sigma^{\prime}}\right)-\lambda_{1} \cdot E_{\sigma}\right)=\left(d-\lambda_{1}\right) F_{Q} .
$$

Notiamo che contraendo la retta per $A$ e $\bar{A}$ in un punto $\mathcal{O} \in Q$, abbiamo creato un nuovo punto base, che è reale, e di molteplicità $d-2 \lambda_{1}$.

Seconda mossa. Sia $B$ un punto base di $\mathscr{H}_{1}$ di maggiore molteplicità $\lambda_{2}$. Esso può essere sia infinitamente vicino a $A$, sia un punto ordinario di $\mathbb{P}^{2}$; in ogni caso, è un punto ordinario di $Q$. Sia $\pi=\pi_{B}: T \rightarrow Q$ lo scoppiamento di $B$ e $\bar{B}$. Si ha

$$
H_{2}=\pi^{*}\left(H_{1}\right)-\lambda_{2} \cdot E_{\pi}=\left(d-\lambda_{1}\right) \cdot \underbrace{\pi^{*}\left(F_{Q}\right)}_{=F_{T}+E_{\pi}}-\lambda_{2} \cdot E_{\pi}=\left(d-\lambda_{1}\right) \cdot F_{T}+\left(d-\lambda_{1}-\lambda_{2}\right) \cdot E_{\pi} .
$$

Ora

$$
K_{T}=\pi^{*}\left(K_{Q}\right)+E_{\pi}=-2 \pi^{*}\left(F_{Q}\right)+E_{\pi}=-2\left(F_{T}+E_{\pi}\right)+E_{\pi}=-2 F_{T}-E_{\pi}
$$

e quindi

$$
E_{\pi}=-K_{T}-2 F_{T}
$$

e viene

$$
H_{2}=\underbrace{\left(-d+\lambda_{1}+2 \lambda_{2}\right)}_{=r} F_{T}-\underbrace{\left(d-\lambda_{1}-\lambda_{2}\right)}_{=s} K_{T} .
$$

Notiamo che le fibre del fibrato in coniche $\gamma: T \rightarrow \mathbb{P}^{1}$ associato al sistema lineare $\left|F_{T}\right|$, si trasformano, tramite $\sigma \circ\left(\sigma^{\prime}\right)^{-1} \circ \pi$, in coniche di $\mathbb{P}^{2}$ che passano per $A, \bar{A}$, $B, \bar{B}$. 
Terza mossa. Sia $C$ un punto base di $\mathscr{H}_{2}$ di maggiore molteplicità $\lambda_{3}$; è un punto ordinario di $T$, e $\bar{C}$ non può giacere sulla stessa fibra di $T$ per la fibrazione in coniche $\gamma: T \rightarrow \mathbb{P}^{1}$; se no, la conica $q \subset \mathbb{P}^{2}$, trasformata per $\sigma \circ\left(\sigma^{\prime}\right)^{-1} \circ \pi$ di una tale fibra, si staccherebbe dalle curve di $\mathscr{H}_{\psi}$. Difatti, $q$ passerebbe per $A, \bar{A}, B, \bar{B}, C, \bar{C}$, e quindi, secondo l'ineguaglianza di Noether, incontrerebbe una curva generica di $\mathscr{H}_{\psi}$ in $2 \cdot\left(\lambda_{1}+\lambda_{2}+\lambda_{3}\right)>2 \cdot(4 m+1)$ punti.

Definiamo $\varphi_{3}$ col triangolo birazionale

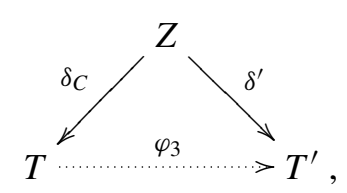

dove $\delta=\delta_{C}$ è lo scoppiamento di $C$ e $\bar{C}$, e $\delta^{\prime}$ la contrazione delle trasformate su $T$ delle due fibre di $\gamma$ che passano per $C$ e $\bar{C}$ : difatti queste sono disgiunte e di self-intersezione -1 . Qui viene

$$
\begin{gathered}
K_{Z}=\delta^{*}\left(K_{T}\right)+E_{\delta}, \quad K_{T^{\prime}}=\delta_{*}^{\prime}\left(K_{Z}\right)=\delta_{*}^{\prime}\left(\delta^{*}\left(K_{T}\right)+E_{\delta}\right)=\delta_{*}^{\prime}\left(\delta^{*}\left(K_{T}\right)\right)+2 F_{T^{\prime}} \\
\delta_{*}^{\prime}\left(\delta^{*}\left(F_{T}\right)\right)=F_{T^{\prime}}
\end{gathered}
$$

e quindi

$$
\begin{aligned}
H_{3} & =\delta_{*}^{\prime}\left(\delta^{*}\left(H_{2}\right)-\lambda_{3} E_{\delta}\right) \\
& =\delta_{*}^{\prime}\left(\delta^{*}\left(r F_{T}-s K_{T}\right)-2 \lambda_{3} F_{T^{\prime}}\right. \\
& =r F_{T^{\prime}}-s\left(K_{T^{\prime}}-2 F_{T^{\prime}}\right)-2 \lambda_{3} F_{T^{\prime}} \\
& =\left(r+2 s-2 \lambda_{3}\right) F_{T^{\prime}}-s K_{T^{\prime}} \\
& =\left(-d+\lambda_{1}+2 \lambda_{2}+2 d-2 \lambda_{1}-2 \lambda_{2}-2 \lambda_{3}\right) F_{T^{\prime}}-\left(d-\lambda_{1}-\lambda_{2}\right) K_{T^{\prime}} \\
& =\underbrace{\left(d-\lambda_{1}-2 \lambda_{3}\right)}_{=t} F_{T^{\prime}}-\underbrace{\left(d-\lambda_{1}-\lambda_{2}\right)}_{=s} K_{T^{\prime}} .
\end{aligned}
$$

Quarta mossa. Osserviamo che $T^{\prime}$ è, come $T$, un fibrato in coniche con due fibre singolari costituite da due fibre immaginarie coniugate, e che $\operatorname{dim}\left(\mathcal{N}_{1}\left(T^{\prime}\right)\right)=$ $\operatorname{dim}\left(\mathcal{N}_{1}(T)\right)=2$. Quindi segue dal iii) della proposizione del $\S 3$ che esiste un morfismo $\pi^{\prime}: T^{\prime} \rightarrow Q$ che presenta $T^{\prime}$ come scoppiamento di due punti immaginari coniugati di $Q$ e

$$
\pi_{*}^{\prime}\left(F_{T}\right)=F_{Q}, \quad \pi_{*}^{\prime}\left(K_{T}\right)=-2 F_{Q}
$$

cosicché

$$
H_{4}=\pi_{*}^{\prime}\left(H_{3}\right)=t F_{Q}+2 s F_{Q}=(t+2 s) F_{Q}=\underbrace{\left(3 d-3 \lambda_{1}-2 \lambda_{3}-2 \lambda_{2}\right)}_{=u} F_{Q} .
$$


Vol. 80 (2005)

Quinta ed ultima mossa. Qui si fa il movimento contrario a quello della prima mossa:

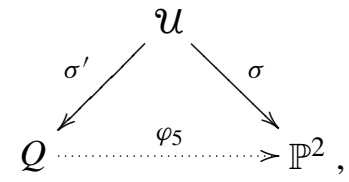

dove $\sigma^{\prime}$ è lo scoppiamento del punto $\mathcal{O}^{\prime}$, immagine di $\mathcal{O}$ per $\varphi_{4} \circ \varphi_{3} \circ \varphi_{2}$, e $\sigma$ contrae il trasformato stretto delle due rette di $Q$ che passano per $\mathcal{O}^{\prime}$.

Notiamo che $\sigma^{\prime *}\left(F_{Q}\right)=E_{\sigma}+2 E_{\sigma^{\prime}}$ e che $\sigma_{*}\left(E_{\sigma^{\prime}}\right)=F_{\mathbb{P}^{2}}$. Viene

$$
\begin{aligned}
H_{5} & =\sigma_{*}\left(\sigma^{*}\left(H_{4}\right)-\operatorname{molt}\left(\mathcal{O}^{\prime}\right) E_{\sigma^{\prime}}\right) \\
& =\sigma_{*}\left(u\left(E_{\sigma}+2 E_{\sigma^{\prime}}\right)-\operatorname{molt}\left(\mathcal{O}^{\prime}\right) E_{\sigma^{\prime}}\right)=\left(2 u-\operatorname{molt}\left(\mathcal{O}^{\prime}\right)\right) F_{\mathbb{P}^{2}}
\end{aligned}
$$

dove $\operatorname{molt}\left(\mathcal{O}^{\prime}\right)=\operatorname{molt}\left(\mathcal{O}^{\prime}, \mathscr{H}_{4}\right)$ denota la molteplicità di una curva generica di $\mathscr{H}_{4}$ in $\mathcal{O}^{\prime}$; poiché intorno a $\mathcal{O}$ il sistema $\mathscr{H}_{1}$ non viene modificato, si ha $\operatorname{molt}\left(\mathcal{O}^{\prime}, \mathscr{H}_{4}\right)=$ $\operatorname{molt}\left(\mathcal{O}, \mathscr{H}_{1}\right)=d-2 \lambda_{1}$, e quindi

$$
H_{5}=\left(6 d-6 \lambda_{1}-4 \lambda_{2}-4 \lambda_{3}-\left(d-2 \lambda_{1}\right)\right) F_{\mathbb{P}^{2}}=\left(d-4\left(\lambda_{1}+\lambda_{2}+\lambda_{3}-d\right)\right) F_{\mathbb{P}^{2}} .
$$

Secondo il lemma del $\S 2$ si ha $\lambda_{1}+\lambda_{2}+\lambda_{3}>d$, e quindi l'ordine di $\psi_{5}$ è inferiore o uguale a $d-4$.

In fin dei conti, l'espressione del grado di $\psi_{5}$, ponendo $d=4 m+1$, è la stessa che quella ottenuta col calcolo che abbiamo fatto per incoraggiarci dopo il lemma del $\S 2$, che era valida solo nel caso in cui i punti di massima molteplicità erano veri punti di $\mathbb{P}^{2}$.

4.2. Diffeomorfismi birazionali di ordine 5. Analizziamo ora il diffeomorfismo birazionale $\varphi=\varphi_{5} \circ \cdots \circ \varphi_{1}$. Nelle mosse del $\S 4.1$, abbiamo utilizzato che $\psi$ non era un isomorfismo lineare solo per assicurarci, nella terza mossa, che i punti $C$ e $\bar{C}$ non fossero su una stessa fibra di $\gamma: T \rightarrow \mathbb{P}^{1}$; quindi possiamo applicare la formula (5) qui sopra nel caso $d=1, \lambda_{1}=\lambda_{2}=\lambda_{3}=0$, per dedurne che $\varphi^{-1}$ è di ordine 5 , e dunque pure $\varphi$ è di ordine 5. La stessa formula, con $d=5$, mostra che ogni trasformazione di ordine cinque è ottenuta in questo modo.

Ragionando con le uguaglianze (1)' e (2) del § 2 e l'ineguaglianza di Noether, vediamo ora che le curve di $\mathscr{H}_{\varphi}$ possiedono 3 paia di punti base di molteplicità 2 . Infatti, se i punti base sono $P_{1}, \ldots, P_{k}$, con molteplicità $\lambda_{1} \geq \cdots \geq \lambda_{k}$, ed i loro coniugati, si ha

$$
\sum \lambda_{i}=6, \quad \sum \lambda_{i}^{2}=12, \quad \lambda_{1}+\lambda_{2}+\lambda_{3} \geq 6
$$

Ne segue che $k=3$ e $\lambda_{1}+\lambda_{2}+\lambda_{3}=6$. Segue allora da $\lambda_{1}^{2}+\lambda_{2}^{2}+\lambda_{3}^{2}=12$ che $\lambda_{1}=\lambda_{2}=\lambda_{3}=2$ (per esempio: almeno una delle $\lambda_{i}$, diciamo $\lambda_{3}$ è pari $\mathrm{e}$ $\lambda_{3}^{2}<12 \Rightarrow \lambda_{3}=2$; quindi $\lambda_{1}^{2}+\lambda_{2}^{2}=8 \Rightarrow \lambda_{1}=\lambda_{2}=2$ ). 
Possiamo anche vedere che $\lambda_{1}=\lambda_{2}=\lambda_{3}=2$ seguendo direttamente le mosse della dimostrazione del $\S 4.1$. Chiamiamo $C^{\prime}, \bar{C}^{\prime}$ i due punti di $T^{\prime}$ immagini delle due fibre di $\gamma: T \rightarrow \mathbb{P}^{1}$ che passano per $C$ e $\bar{C}$ rispettivamente; $B^{\prime}, \bar{B}^{\prime}$ i due punti di $Q$ immagini del divisore eccezionale $E_{\pi^{\prime}}$ di $\pi^{\prime}$ e $A^{\prime}, \bar{A}^{\prime}$ i punti di $\mathbb{P}^{2}$ immagini del divisore $E^{\prime}$ di bigrado $(1,1)$ di $Q$ costituito dalle due rette che passano per $\mathcal{O}^{\prime}$ : queste 3 coppie costituiscono i punti base di $\varphi^{-1}$. Deduciamo ora dai calcoli del paragrafo precedente che la molteplicità di $\mathscr{H}_{\varphi^{-1}}$ in questi punti è uguale a 2. Difatti, per $\psi=$ identità, utilizzando (2) con $d=1, \lambda_{1}=\lambda_{2}=0$ abbiamo

$$
\operatorname{molt}\left(C^{\prime}, \mathcal{H}_{\psi_{3}}\right)=\delta^{*}\left(H_{2}\right) \cdot\left(\delta^{*}\left(F_{T}\right)-E_{\delta}\right)=H_{2} \cdot F_{T}=\left(-F_{T}-K_{T}\right) \cdot F_{T}=2 .
$$

Ora utilizziamo (3) con $d=1, \lambda_{1}=\lambda_{2}=\lambda_{3}=0$ :

$$
2 \operatorname{molt}\left(B^{\prime}, \mathscr{H}_{\psi_{4}}\right)=H_{3} \cdot E_{\pi^{\prime}}=\left(F_{T^{\prime}}-K_{T^{\prime}}\right) \cdot\left(-K_{T^{\prime}}-2 F_{T^{\prime}}\right)=4 .
$$

Infine, con (4) e $d=1, \lambda_{1}=\lambda_{2}=\lambda_{3}=0$ :

$$
\begin{aligned}
2 \operatorname{molt}\left(A^{\prime}, \mathcal{H}_{\psi_{5}}\right) & =\left(\sigma^{\prime *}\left(H_{4}\right)-E_{\sigma^{\prime}}\right) \cdot\left(\sigma^{\prime *}\left(F_{Q}\right)-2 E_{\sigma^{\prime}}\right) \\
& =H_{4} \cdot F_{Q}+2 E_{\sigma^{\prime}} \cdot E_{\sigma^{\prime}}=3 F_{Q}^{2}+2 E_{\sigma^{\prime}}^{2}=4 .
\end{aligned}
$$

Osserviamo che l'unica condizione sui punti $A, B$ e $C$ è che essi ed i loro coniugati non giacciano su una stessa conica, il che viene a dire che $C$ e $\bar{C}$ non giacciano su una stessa fibra di $\gamma$ (vedere la terza mossa del paragrafo precedente). Possono presentarsi tre casi.

1) La $\varphi$ ha 3 punti base distinti su $\mathbb{P}^{2}$ e i loro coniugati, con molteplicità due. Questo è il caso in cui $B$ non giace su $\sigma^{\prime}\left(E_{\sigma}\right)$, e scriviamo, per semplificare, $B$ per $\sigma\left(\sigma^{\prime-1}(B)\right)$. Come pure, $C$ non giace su $E_{\pi}$, né $\pi(C)$ su $\sigma^{\prime}\left(E_{\sigma}\right)$, e scriviamo $C$ per $\sigma\left(\sigma^{\prime-1}(\pi(C))\right)$. Conformemente alla notazione introdotta nel $\S 1$, il sistema lineare $\mathcal{H}_{\varphi}$ associato a $\varphi$ è

$$
\mathcal{C}^{5}\left(A^{2}, \bar{A}^{2}, B^{2}, \bar{B}^{2}, C^{2}, \bar{C}^{2}\right)
$$

che, come nel $\S 1$, scriveremo più semplicemente $\mathscr{D}^{5}\left(A^{2}, B^{2}, C^{2}\right)$. La curva generica del sistema è una quintica con punti doppi ordinari in $A, B, C$ (ed i loro coniugati). L'insieme di queste $\varphi$ è stato designato con $\Phi_{5}$.

2) La $\varphi$ ha due punti base distinti $A$ e $C$ su $\mathbb{P}^{2}$ e un punto base $B$ infinitamente prossimo. Allora $B$ appartiene a $\sigma^{\prime}\left(E_{\sigma}\right)$, ma $C \notin E_{\pi}$; notiamo che in tal caso $\pi(C) \notin \sigma^{\prime}\left(E_{\sigma}\right)$, se no, come abbiamo visto innanzi nella terza mossa, si staccherebbe una conica dalle curve di $\mathcal{H}_{\varphi}$. Scriviamo ancora $C$ per $\sigma\left(\sigma^{\prime-1}(\pi(C))\right) \in \mathbb{P}^{2}$. Indichiamo il sistema lineare $\mathscr{H}_{\varphi}$ con

$$
D^{5}\left(A^{2} \leftarrow B^{2}, C^{2}\right)
$$


dove la freccia $A \leftarrow B$ sta a indicare che $B$ è infinitamente prossimo ad $A$. La curva generica del sistema è una quintica con punto doppio ordinario in $C$, ed una tacnode ordinaria con tangente fissa (rappresentata da $B$ ) nel punto $A$.

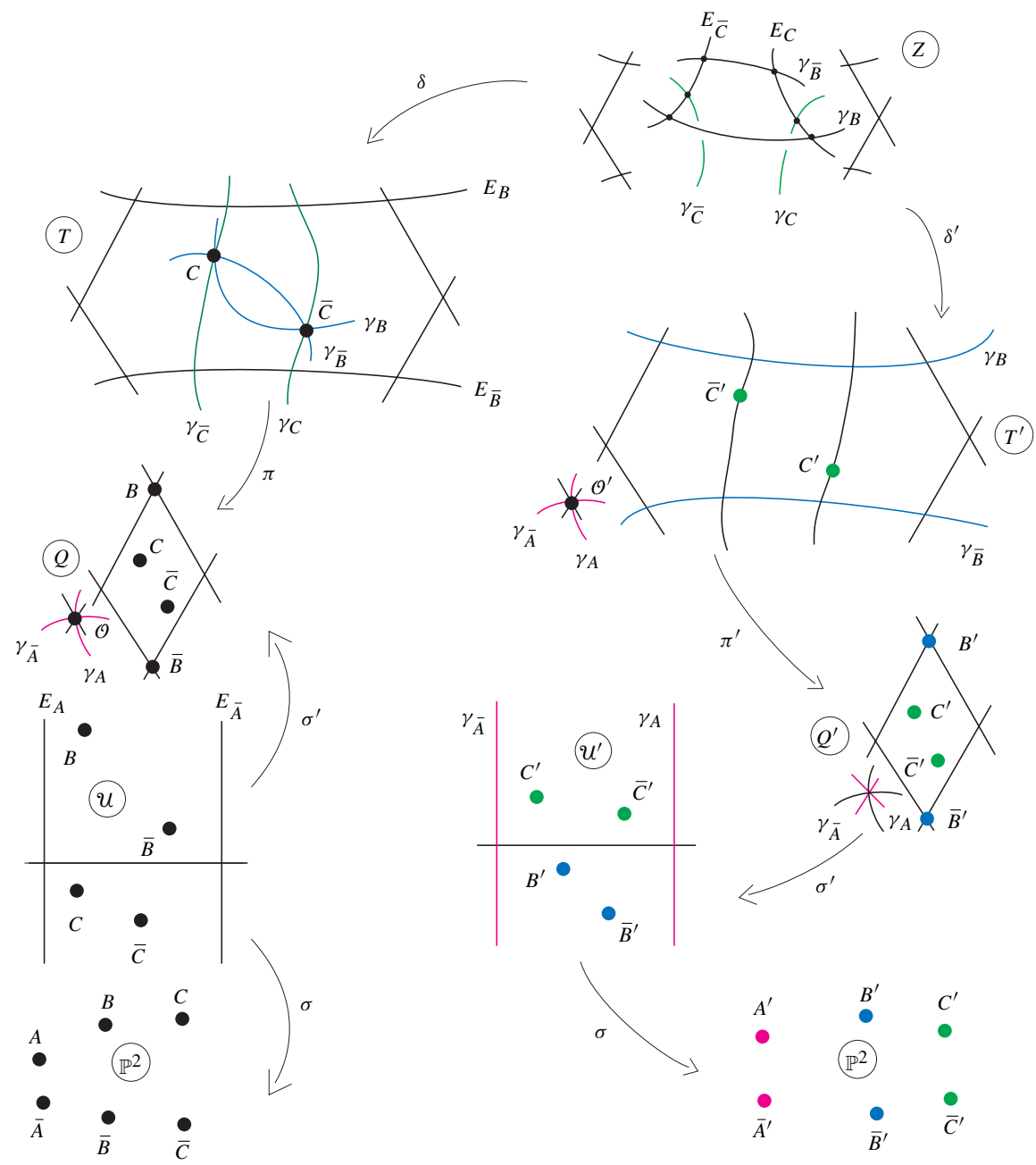

Figura 1. Decomposizione di Sarkisov di $D^{5}\left(A^{2}, B^{2}, C^{2}\right)$.

3) La $\varphi$ ha un solo punto base $A$ su $\mathbb{P}^{2}$; allora $B \in \sigma^{\prime}\left(E_{\sigma}\right)$ e $C \in E_{\pi}$. In questo caso indicheremo il sistema lineare $\mathcal{H}_{\varphi}$ con

$$
D^{5}\left(A^{2} \leftarrow B^{2} \leftarrow C^{2}\right) .
$$

La curva generica è una quintica con tacnode ordinaria in $A$, con tangente fissa (rappresentata da $B$ ) e cerchio osculatore fisso (rappresentato da $C$ ). 
Il caso di $B, \pi(C) \in \sigma^{\prime}\left(E_{\sigma}\right)$ non si può presentare, come abbiamo già visto.

Descriviamo le giacobiane di queste trasformazioni:

1) $D^{5}\left(A^{2}, B^{2}, C^{2}\right)$. Chiamiamo $\gamma_{A}, o$ o $\gamma_{A}^{\varphi}$ se occorre precisare, la conica per tutti i punti base salvo $A$, ed in modo simile $\gamma_{\bar{A}}, \gamma_{B}, \gamma_{\bar{B}}, \gamma_{C}, \gamma_{\bar{C}}$. Ognuna di queste sei coniche incontra la quintica generica $\theta \in D^{5}\left(A^{2}, B^{2}, C^{2}\right)$ in cinque punti doppi, che sono i punti base sulla conica; quindi, se $\theta$ incontra la conica fuori dai punti base, allora contiene la conica. Come abbiamo già visto al $\S 1$, ne segue che, se $\varphi: \mathbb{P}^{2} \quad \cdots>\mathbb{P}^{2}$ è la trasformazione ottenuta scegliendo una base di $D^{5}\left(A^{2}, B^{2}, C^{2}\right)$, essa schiaccia le sei coniche su sei punti $A^{\prime}=\varphi\left(\gamma_{A}\right), \bar{A}^{\prime}=\varphi\left(\gamma_{\bar{A}}\right)$, e via di seguito; questi saranno i punti base di $\varphi^{-1}$. Le sei coniche costituiscono la giacobiana di $\varphi$.

Seguiamo ora come la giacobiana di $\varphi$ si trasforma secondo le mosse del $\S 4.1$. $\mathrm{Ci}$ aiuteremo con la figura 1, nella quale $E_{X}$ indica in generale la fibra eccezionale dello scoppiamento di $X$, e $L_{A, \bar{A}}$ indica la retta per $A$ e $\bar{A}$; si userà la stessa lettera per una curva (o un punto) e il suo trasformato stretto sui vari spazi.

Sia $\mathbb{P}_{A, B}^{1}$ il fascio delle coniche per $A, \bar{A}, B, \bar{B}$. Si definisce un morfismo razionale $\gamma: \mathbb{P}^{2} \quad \cdots .>\mathbb{P}_{A, B}^{1}$ associando a $X \in \mathbb{P}^{2}$ la conica per $X, A, \bar{A}, B, \bar{B}$; come abbiamo già osservato in 4.1, seconda mossa, questo diventa un vero morfismo $\gamma: T \rightarrow \mathbb{P}_{A, B}^{1}$, che è un fibrato in coniche, con due fibre singolari su $L_{A, \bar{B}} \cup L_{\bar{A}, B}$ e $L_{A, B} \cup L_{\bar{A}, \bar{B}}$.

Per passare da $T$ a $T^{\prime}$, si fanno scoppiare $C$ e $\bar{C}$ e si contraggono $\gamma_{C}$ e $\gamma_{\bar{C}}$. Si ha ancora un fibrato $\gamma^{\prime}: T^{\prime} \rightarrow \mathbb{P}_{A, B}^{1}$, solo che le due fibre $\gamma_{C}$ e $\gamma_{\bar{C}}$ sono state modificate. Su $T^{\prime}$ la conica $\gamma_{B}$ taglia ogni fibra di $\gamma^{\prime}$ in esattamente un punto, quindi $\gamma_{B}$ è una sezione di $\gamma^{\prime}$, come pure la coniugata $\gamma_{\bar{B}}$. Quindi, seguendo il iii) della proposizione del $\S 3$, comprimendo $\gamma_{B}$ e $\gamma_{\bar{B}}$ si passa da $T^{\prime}$ a un $Q^{\prime}$ isomorfo a $Q$.

Passando da $\mathbb{P}^{2}$ a $Q$, si contrae la retta $L_{A, \bar{A}}$ in un punto $\mathcal{O}$; le coniche $\gamma_{A}$ e $\gamma_{\bar{A}}$ sono le uniche componenti della giacobiana le cui trasformate passano per $\mathcal{O}$, il cui trasformato su $Q^{\prime}$ è chiamato $\mathcal{O}^{\prime}$. Passando da $Q^{\prime}$ a $\mathbb{P}^{2}$, si fa prima scoppiare $\mathcal{O}^{\prime}$, e poi si contraggono $\gamma_{A}$ e $\gamma_{\bar{A}}$.

2) $D^{5}\left(A^{2} \leftarrow B^{2}, C^{2}\right)$. Procedendo come prima, qui si può vedere che la giacobiana è composta dalle 4 coniche $\gamma_{B}, \gamma_{\bar{B}}, \gamma_{C}, \gamma_{\bar{C}}$; le coniche $\gamma_{B}$ e $\gamma_{\bar{B}}$ appaiono doppie nella giacobiana, perché sono limite dell'unione di $\gamma_{A}$ e $\gamma_{B}$ (rispettivamente $\gamma_{\bar{A}}$ e $\gamma_{\bar{B}}$ ) del caso 1), quando si fa tendere il punto $B$ verso $A$.

La decomposizione di Sarkisov è simile al caso 1), solo che ora $\mathcal{O}$ è il punto singolare di una delle due fibre singolari di $\gamma: T \rightarrow \mathbb{P}_{A, B}^{1}$ (quella sulla conica unione delle rette rappresentate da $B$ e $\bar{B}$ ). Seguendo le varie trasformazioni sulla figura 2, si vede che in fin dei conti $B$ e $\bar{B}$ vengono trasformati su due punti $B^{\prime}$ e $\bar{B}^{\prime}$ infinitamente prossimi a $A^{\prime}$ e $\bar{A}^{\prime}$ rispettivamente. 


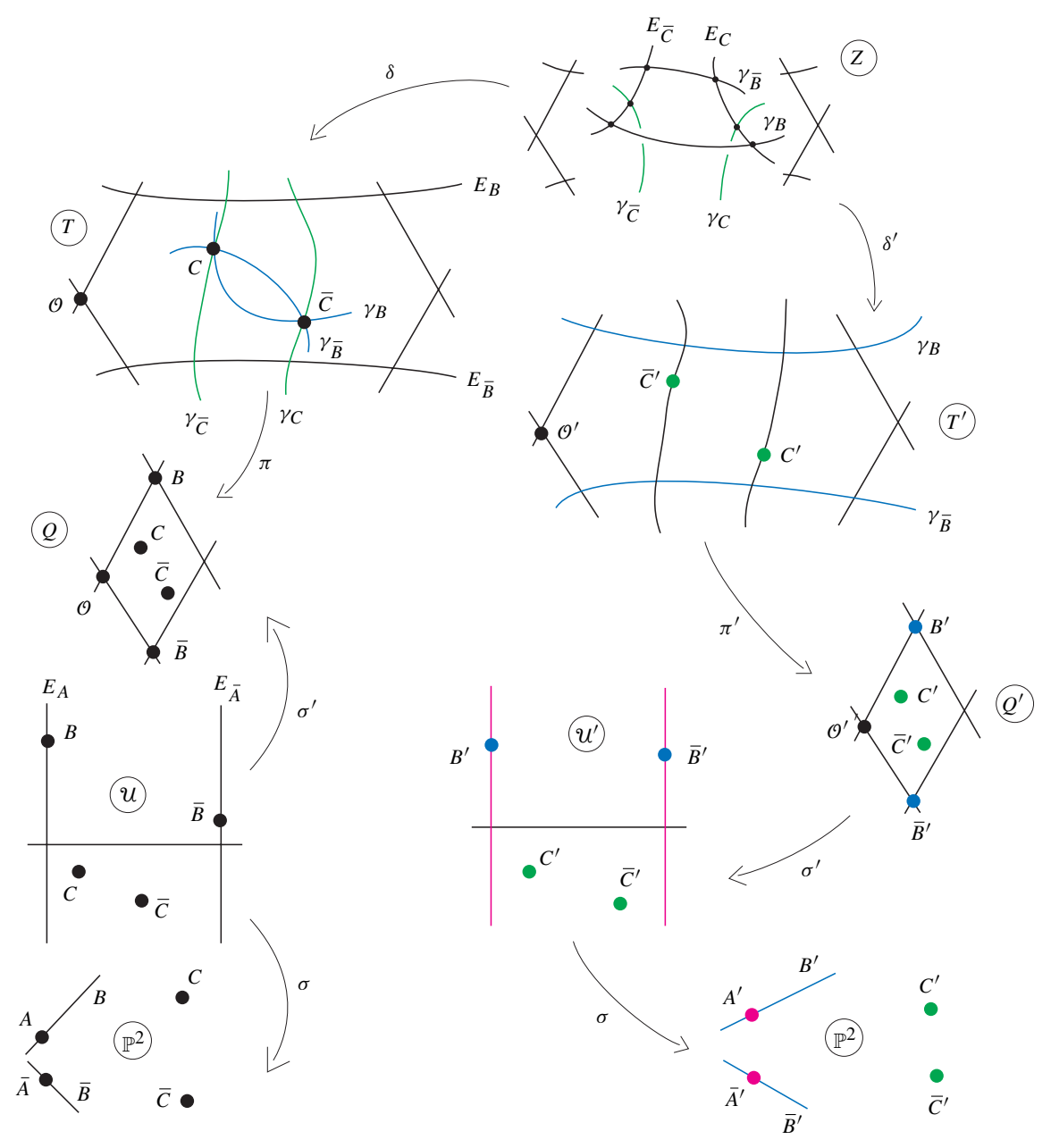

Figura 2. Decomposizione di Sarkisov di $D^{5}\left(A^{2} \leftarrow B^{2}, C^{2}\right)$.

3) Nel caso di $\mathscr{D}^{5}\left(A^{2} \leftarrow B^{2} \leftarrow C^{2}\right)$, ci limitiamo a indicare che la giacobiana è l'unione delle due coniche $\gamma_{C}$ e $\gamma_{\bar{C}}$, ognuna con molteplicità 3 .

Torniamo ora alla prova del teorema II. Occorre dunque dimostrare che le trasformazioni dei casi 2) e 3) si possono scrivere come composizioni di trasformazioni del caso 1), cioè di tipo $\Phi_{5}$. Procederemo così: data una $\psi$ di tipo 2 ) o 3), costruiamo $\varphi \in \Phi_{5}$ tale che $\psi \circ \varphi^{-1}$ sia una trasformazione di grado 9 , che a sua volta scriveremo come prodotto di due trasformazioni di $\Phi_{5}$; questo passaggio per una trasformazione di grado più alto per scomporre la $\psi$ è inevitabile: la composizione di due trasformazioni di $\Phi_{5}$, se non è lineare, è sempre di grado almeno 9. 
Per capire com'è la trasformazione $\psi \circ \varphi^{-1}$, si prende una curva generica $\theta$ di $\mathscr{H}_{\psi}$ e si cerca di capire com'è fatta $\varphi(\theta)$, che sarà la curva generica del sistema $\mathscr{H}_{\psi \circ \varphi^{-1}}$. Qui sarà utile la decomposizione di Sarkisov: $\varphi=\varphi_{5} \circ \cdots \circ \varphi_{1}$, perché ogni $\varphi_{i}$ è notevolmente semplice.

a) $\psi$ è del tipo $\mathscr{D}^{5}\left(A^{2} \leftarrow B^{2}, C^{2}\right)$. Chiameremo $q\left(X_{1}, X_{2}, X_{3}, X_{4}, X_{5}\right)$ la conica per i punti $X_{1}, X_{2}, X_{3}, X_{4}, X_{5}$, e scriveremo $q\left(X_{1}, \ldots, X_{k}\right)$ per indicare che esiste una conica che passa per i punti $X_{1}, \ldots, X_{k}$, e per indicare la conica stessa.

Prendiamo una $\varphi$ associata al sistema lineare $D^{5}\left(A^{2}, C^{2}, D^{2}\right)$, dove il punto $D$ è scelto in modo tale che una conica della giacobiana di $\varphi$ e una della giacobiana di $\psi$ non abbiano punti in comune all'infuori di $A, \bar{A}, C$ e $\bar{C}$, cioè:

- le coniche della giacobiana di $\varphi$ che passano per $A$ non devono essere tangenti alla tangente $B$ della tacnode di $\theta$ in $A$, e quindi le coniche della giacobiana di $\varphi$ che passano per $\bar{A}$ non saranno tangenti a $\bar{B}$;

- $D, \bar{D} \notin q(A, \bar{A}, B, C, \bar{C})=\gamma_{\bar{B}}^{\psi}, D, \bar{D} \notin q(A, \bar{A}, B, \bar{B}, C)=\gamma_{\bar{C}}^{\psi}$ e quindi $D, \bar{D} \notin q(A, \bar{A}, \bar{B}, C, \bar{C})=\gamma_{B}^{\psi}, D, \bar{D} \notin q(A, \bar{A}, B, \bar{B}, \bar{C})=\gamma_{C}^{\psi}$.

Chiamiamo $A^{\prime}, C^{\prime}, D^{\prime}$ e i loro coniugati i punti base di $\varphi^{-1}$, che poi sono le immagini per $\varphi$ di $\gamma_{A}^{\varphi}, \gamma_{C}^{\varphi}, \gamma_{D}^{\varphi}$ e delle loro coniugate.

La quintica generica $\theta$ del sistema $\mathscr{H}_{\psi}$ taglia $\gamma_{A}^{\varphi}$ e $\gamma_{C}^{\varphi}$ in $2 \cdot 5-3 \cdot 2=4$ punti liberi, $\gamma_{D}^{\varphi}$ in $2 \cdot 5-4 \cdot 2=2$ punti liberi. Ne segue che $\varphi(\theta)$ avrà punti quadrupli in $A^{\prime}, C^{\prime}, \bar{A}^{\prime}, \bar{C}^{\prime}$ e punti doppi in $D^{\prime}, \bar{D}^{\prime}$. Ci resta da capire cosa avviene dei punti di $\theta$ che cadono nei punti base di $\psi$ :

- $\operatorname{per} C$ ( e analogamente per $\bar{C}$ ), dato che la tangente ai rami di $\theta$ per questo punto sono variabili, dopo lo scoppiamento di $C$ (seconda mossa), la trasformata di $\theta$ su $T$ taglierà la sezione di $\gamma: T \rightarrow \mathbb{P}^{1}$, cioè ognuna delle due componenti del divisore eccezionale di $\pi: T \rightarrow Q$, in due punti qualsiasi, che verranno infine trasformati in due punti variabili delle coniche $\gamma_{C^{\prime}}^{\varphi^{-1}}$ e $\gamma_{\bar{C}^{\prime}}^{\varphi^{-1}}$ rispettivamente (per seguire le mosse sulla figura 1 , occorre sostituire i punti $B$ e $C$ della figura rispettivamente con i punti $C$ e $D$ di questo paragrafo);

- per $A$ invece, $\theta$ ha due rami tangenti alla stessa retta $B$. La trasformata di $\theta$ su $Q$ passerà quindi doppiamente per i punti $B, \bar{B} \in \sigma^{\prime}\left(E_{\sigma}\right)$, che grazie all'ipotesi di genericità su $D$ e $\bar{D}$ non giacciono né su $\gamma_{A}^{\varphi}$, né su $\gamma_{\bar{A}}^{\varphi}$. Andranno a finire su dei veri punti $G^{\prime}, \bar{G}^{\prime}$ di $\mathbb{P}^{2}$ (che giacciono sulle coniche $\gamma_{A^{\prime}}^{\varphi^{-1}}=$ $q\left(\bar{A}^{\prime}, C^{\prime}, \bar{C}^{\prime}, D^{\prime}, \bar{D}^{\prime}\right)$ e $\gamma_{\bar{A}^{\prime}}^{\varphi^{-1}}=q\left(A^{\prime}, C^{\prime}, \bar{C}^{\prime}, D^{\prime}, \bar{D}^{\prime}\right)$ rispettivamente $)$.

Poiché $\theta$ taglia una curva generica di $D^{5}\left(A^{2}, C^{2}, D^{2}\right)$ in $A, \bar{A}, C, \bar{C}$ con molteplicità $4, \varphi(\theta)$ sarà una curva di grado $5 \cdot 5-4 \cdot 4=9$ (questo segue anche dalla formula (5) del $\S 4.1$, con $d=5, \lambda_{1}=\lambda_{2}=2, \lambda_{3}=0$ ). 
Dunque $\varphi(\theta)$ appartiene al sistema lineare $D^{9}\left(A^{\prime 4}, C^{\prime 4}, D^{\prime 2}, G^{\prime 2}\right)$, dove $A^{\prime}, C^{\prime}$, $D^{\prime}$ sono generici, mentre $G^{\prime}$ deve stare sulla conica $\gamma_{A^{\prime}}^{\varphi^{-1}}$.

Osserviamo che $A^{\prime}, C^{\prime}, G^{\prime}$ ed i loro coniugati non sono su una stessa conica, se no questa si staccherebbe dalle curve di $D^{9}\left(A^{\prime 4}, C^{\prime 4}, D^{\prime 2}, G^{\prime 2}\right)$. Sia dunque $\tau$ una trasformazione di ordine 5 associata al sistema lineare $D^{5}\left(A^{\prime 2}, C^{\prime 2}, G^{2}\right)$, e siano $A^{\prime \prime}, C^{\prime \prime}, G^{\prime \prime}$ i punti base dell'inverso di $\tau$.

Vediamo ora che $D^{\prime}$ e $\bar{D}^{\prime}$ non giacciono sulla giacobiana di $\tau$ :

- $D^{\prime}, \bar{D}^{\prime} \notin \gamma_{G^{\prime}}^{\tau} \cup \gamma_{\bar{G}^{\prime}}^{\tau}:$ Se $D^{\prime} \in \gamma_{G^{\prime}}^{\tau}=q\left(A^{\prime}, \bar{A}^{\prime}, C^{\prime}, \bar{C}^{\prime}, \bar{G}^{\prime}\right)$ o se $\bar{D}^{\prime} \in \gamma_{G^{\prime}}^{\tau}$, allora questa conica si staccherebbe dal $D^{9}\left(A^{\prime 4}, C^{\prime 4}, D^{\prime 2}, G^{\prime 2}\right)$. Quindi né $D^{\prime}$ né $\bar{D}^{\prime}$ appartengono a $\gamma_{G^{\prime}}^{\tau}$ o $\gamma_{\bar{G}^{\prime}}^{\tau}$.

- $D^{\prime}, \bar{D}^{\prime} \notin \gamma_{A^{\prime}}^{\tau} \cup \gamma_{\bar{A}^{\prime}}^{\tau}$ : Se $D^{\prime}$ appartenesse a $q\left(G^{\prime}, \bar{G}^{\prime}, C^{\prime}, \bar{C}^{\prime}, A^{\prime}\right)=\gamma_{\bar{A}^{\prime}}^{\tau}$, poiché la conica $q\left(A^{\prime}, C^{\prime}, \bar{C}^{\prime}, D^{\prime}, \bar{D}^{\prime}\right)=\gamma_{\bar{A}^{\prime}}^{\varphi^{-1}}$ contiene anche $\bar{G}^{\prime}$, queste due coniche avrebbero in comune i cinque punti $A^{\prime}, C^{\prime}, \bar{C}^{\prime}, D^{\prime}, \bar{G}^{\prime}$ e quindi coinciderebbero. Ma allora $\gamma_{\bar{A}^{\prime}}^{\tau}$ dovrebbe contenere $C^{\prime}, D^{\prime}, G^{\prime}$ ed i loro coniugati, e sarebbe quindi una conica reale, componente fissa di $\mathscr{H}_{\varphi^{-1}}$.

Se $D^{\prime}$ appartenesse a $q\left(G^{\prime}, \bar{G}^{\prime}, C^{\prime}, \bar{C}^{\prime}, \bar{A}^{\prime}\right)$, ragionando come prima, si vede che $\gamma_{A^{\prime}}^{\tau}$ sarebbe una componente fissa di $\mathscr{H}_{\varphi^{-1}}$.

- $D^{\prime}, \bar{D}^{\prime} \notin \gamma_{C^{\prime}}^{\tau} \cup \gamma_{\bar{C}^{\prime}}^{\tau}:$ Se $D^{\prime} \in \gamma_{\bar{C}^{\prime}}^{\tau}$, poniamo $\gamma=\gamma_{\bar{C}^{\prime}}^{\tau}=q\left(A^{\prime}, \bar{A}^{\prime}, C^{\prime}, G^{\prime}, \bar{G}^{\prime}\right)=$ $q\left(A^{\prime}, \bar{A}^{\prime}, C^{\prime}, G^{\prime}, \bar{G}^{\prime}, D^{\prime}\right)$. Vogliamo far vedere che $\varphi^{-1}(\gamma)=q(A, \bar{A}, B, \bar{B}, C, D)$ in questo caso, in contraddizione con l'ipotesi di genericità posta su $D$.

Notiamo che $\gamma$ non contiene né $\bar{C}^{\prime}$, né $\bar{D}^{\prime}$, se no $\gamma$ sarebbe reale, quindi $\gamma=$ $q\left(A^{\prime}, \bar{A}^{\prime}, C^{\prime}, \bar{C}^{\prime}, G^{\prime}, \bar{G}^{\prime}, D^{\prime}, \bar{D}^{\prime}\right)$ sarebbe componente fissa del $D^{9}\left(A^{\prime 4}, C^{\prime 4}, D^{\prime 2}, G^{\prime 2}\right)$.

L'intersezione di $\gamma$ e $\gamma_{A^{\prime}}^{\varphi^{-1}}=q\left(\bar{A}^{\prime}, C^{\prime}, \bar{C}^{\prime}, D^{\prime}, \bar{D}^{\prime}\right)$ contiene i tre punti base $\bar{A}^{\prime}, C^{\prime}, D^{\prime}$, ma non contiene né $\bar{C}^{\prime}$, né $\bar{D}^{\prime}$. Quindi $\gamma \cap \gamma_{A^{\prime}}^{\varphi^{-1}}$ contiene esattamente un punto libero, e allora $\varphi^{-1}(\gamma) \ni A$.

Con ragionamenti simili, si vede che $\varphi^{-1}(\gamma) \ni \bar{A}, C, D$ e quindi avremmo appunto $q(A, \bar{A}, B, \bar{B}, C, D)$.

Se $\bar{D}^{\prime} \in \gamma=\gamma_{\bar{C}^{\prime}}^{\tau}$ si procede in modo simile; qui $\gamma=q\left(A^{\prime}, \bar{A}^{\prime}, C^{\prime}, G^{\prime}, \bar{G}^{\prime}, \bar{D}^{\prime}\right)$, e $D^{\prime}, \bar{C}^{\prime} \notin \gamma$. Se ne dedurrebbe che $\varphi^{-1}(\gamma)=q(A, \bar{A}, B, \bar{B}, C, \bar{D})$, contrariamente all'ipotesi posta su $D$.

Ne deduciamo che $\tau\left(D^{\prime}\right)$ è un vero punto di $\mathbb{P}^{2}$, distinto da $A^{\prime \prime}$ e $B^{\prime \prime}$. Procedendo come per l'esame di $\varphi\left(D^{5}\left(A^{2} \leftarrow B^{2}, C^{2}\right)\right)$, si vede che

$$
\tau\left(D^{9}\left(A^{\prime 4}, C^{\prime 4}, D^{\prime 2}, G^{\prime 2}\right)\right)=\mathscr{D}^{5}\left(A^{\prime \prime 2}, C^{\prime \prime 2}, \tau\left(D^{\prime}\right)^{2}\right) .
$$


In fin dei conti, abbiamo mostrato che $\psi \circ \varphi^{-1} \circ \tau^{-1} \in \Phi_{5}$.

È da notare che il sistema lineare $\mathcal{D}^{9}\left(A^{\prime 4}, C^{\prime 4}, D^{\prime 2}, G^{\prime 2}\right)$ associato a $\xi=\psi \circ \varphi^{-1}$, benché abbia punti base con singolarità ordinarie, non è generico, perché $G^{\prime}$ e $\bar{G}^{\prime}$ stanno su $\gamma_{A^{\prime}}^{\varphi^{-1}}$ e $\gamma_{\bar{A}^{\prime}}^{\varphi^{-1}}$ rispettivamente, come abbiamo visto. Studiando l'immagine per $\psi$ di una curva del sistema di $\varphi$, chiamando $A^{*}, B^{*}, C^{*}$ i punti base di $\psi^{-1}$, si può vedere che le curve del sistema lineare di $\xi^{-1}$ appartengono a $D^{9}\left(A^{* 4} \leftarrow\right.$ $\left.B^{* 2}, C^{* 4}, \psi(D)^{2}\right)$, cioè possiedono in $A^{*}$ un punto quadruplo, nel quale due rami hanno una tangente fissa, rappresentata da $B^{*}$.

b) $\psi \grave{e ̀ ~ d e l ~ t i p o ~} \mathscr{D}^{5}\left(A^{2} \leftarrow B^{2} \leftarrow C^{2}\right)$. Qui possiamo servirci del caso precedente, cioè utilizzare, per semplificare $\psi$, trasformazioni $\varphi$ di tipo $D^{5}\left(A^{2} \leftarrow B^{2}, D^{2}\right)$, con $D$ generico, cioè
$C, \bar{C} \notin \gamma_{D}^{\varphi} \cup \gamma_{\bar{D}}^{\varphi}$
(2) $C, \bar{C} \notin \gamma_{B}^{\varphi} \cup \gamma_{\bar{B}}^{\varphi}$
(3)
$D, \bar{D} \notin \gamma_{C}^{\psi} \cup \gamma_{\bar{C}}^{\psi}$

Una curva generica di $\mathscr{H}_{\varphi}$ è una quintica con punti doppi in $D, \bar{D}$, e tacnodi in $A, \bar{A}$, con tangente fissa uguale a $B, \bar{B}$, cioè la stessa tangente che per la curva generica del sistema lineare $\mathscr{H}_{\psi}$. Sia $\mathscr{H}_{\varphi^{-1}}=\mathscr{D}^{5}\left(A^{\prime 2} \leftarrow B^{\prime 2}, D^{\prime 2}\right)$.

Osservazione. Sia $\theta$ una qualsiasi curva che taglia $\gamma_{B}^{\varphi}$ in un punto $X$, fuori dai punti base di $\varphi$, e consideriamo solo i punti di $\theta$ in un intorno di $X$. Il trasformato di $\theta$ su $Q^{\prime}$ passerà per $B^{\prime}$, e quindi $\varphi(\theta)$ sarà tangente in $A^{\prime}$ alla retta fissa di $\mathbb{P}^{2}$ rappresentata da $B^{\prime}$.

Se ora $\theta$ è la curva generica di $D^{5}\left(A^{2} \leftarrow B^{2} \leftarrow C^{2}\right)$, essa taglierà $\gamma_{B}^{\varphi}$ in 4 punti liberi e $\gamma_{D}^{\varphi}$ in due punti liberi (utilizziamo le proprietà di genericità (1) e (2) poste su $D)$. Ne segue che $\varphi(\theta)$ avrà in $A^{\prime}$ un punto quadruplo con quattro rami tutti tangenti alla retta per $A^{\prime}$ rappresentata da $B^{\prime}$ (e lo stesso tipo di singolarità in $\bar{A}^{\prime}$ ), e avrà un punto doppio ordinario in $D^{\prime}=\varphi\left(\gamma_{D}^{\varphi}\right)$ (e simile in $\bar{D}^{\prime}$ ).

Per seguire le mosse sulla figura 2 , occorre sostituire il punto $C$ della figura con il punto $D$ di questo paragrafo. Resta da vedere cosa avviene nel punto base $A$ (e analogamente $\bar{A}$ ); su $U$, il punto doppio con due rami tangenti di uguale curvatura di $\theta$ si trasforma in una tacnode ordinaria per $B$, e su $T$ diventa un punto doppio ordinario in $C$, con tangenti ai rami libere, ed infine nel $\mathbb{P}^{2}$ di arrivo, sarà un punto doppio ordinario $G^{\prime}$, con tangenti ai rami libere (utilizziamo (1)), che giace su $\gamma_{B^{\prime}}^{\varphi^{-1}}=$ $q\left(A^{\prime}, \bar{A}^{\prime}, \bar{B}^{\prime}, D^{\prime}, \bar{D}^{\prime}\right)$. Ne segue che

$$
\varphi\left(D^{5}\left(A^{2} \leftarrow B^{2} \leftarrow C^{2}\right)\right)=\mathscr{D}^{9}\left(A^{\prime 4} \leftarrow B^{\prime 4}, D^{\prime 2}, G^{\prime 2}\right)
$$

e i punti $\left(A^{\prime} \leftarrow B^{\prime}, D^{\prime}\right)$ non giacciono su una stessa conica, e neppure i punti $\left(A^{\prime} \leftarrow\right.$ $\left.B^{\prime}, G^{\prime}\right)$, se no si staccherebbero dal $D^{9}\left(A^{\prime 4} \leftarrow B^{\prime 4}, D^{\prime 2}, G^{\prime 2}\right)$, mentre $G^{\prime} \in \gamma_{B^{\prime}}^{\varphi^{-1}}$, $\bar{G}^{\prime} \in \gamma_{\bar{B}^{\prime}}^{\varphi^{-1}}$. 
Consideriamo ora la trasformazione $\chi$ d'ordine 5 associata al sistema lineare $D^{5}\left(A^{\prime 2} \leftarrow B^{\prime 2}, G^{\prime 2}\right)$; sia $\theta \in D^{9}\left(A^{\prime 4} \leftarrow B^{\prime 4}, D^{\prime 2}, G^{\prime 2}\right)$. Poiché $\theta$ taglia una curva generica del $D^{5}\left(A^{\prime 2} \leftarrow B^{\prime 2}, G^{\prime 2}\right)$ in $A^{\prime}, \bar{A}^{\prime}$ con molteplicità 16 , e in $G^{\prime}, \bar{G}^{\prime}$ con molteplicità $4, \chi(\theta)$ sarà di grado $5 \cdot 9-2 \cdot 16-2 \cdot 4=5$.

Nella decomposizione di Sarkisov di $\chi$, la trasformata di $\theta$ su $Q$ ha due punti quadrupli ordinari in $B^{\prime}, \bar{B}^{\prime}$, che corrispondono a $A^{\prime}$ e $\bar{A}^{\prime}$, in più delle due paia di punti doppi in $D^{\prime}, \bar{D}^{\prime}$ e $G^{\prime}, \bar{G}^{\prime}$. Passando a $T$, i due punti quadrupli diventano 4 punti ordinari di $E_{B^{\prime}}$ e $E_{\bar{B}^{\prime}}$ rispettivamente, e finiscono come punti ordinari del $\mathbb{P}^{2}$ di arrivo. Il destino del paio di punti doppi $G^{\prime}, \bar{G}^{\prime}$ è simile.

I punti doppi $D^{\prime}$ e $\bar{D}^{\prime}$ non sono punti base di $\chi$, e procedendo come nel caso b), utilizzando la proprietà di genericità (3) posta su $D$ e $q\left(A^{\prime}, \bar{A}^{\prime}, \bar{B}^{\prime}, D^{\prime}, \bar{D}^{\prime}, G^{\prime}\right)$, si vede che $D$ e $D^{\prime}$ non giacciono sulla giacobiana di $\chi$. Le loro immagini sono due punti doppi $D^{\prime \prime}=\chi\left(D^{\prime}\right)$ e $\bar{D}^{\prime \prime}=\chi\left(\bar{D}^{\prime}\right)$.

L'intersezione di $\theta$ con $\gamma_{G^{\prime}}^{\chi}$ contiene $A^{\prime}, \bar{A}^{\prime}, B^{\prime}$ e $\bar{B}^{\prime}$ con molteplicità 4 , più $\bar{G}^{\prime}$ con molteplicità 2 ; in tutto contano per $4 \cdot 4+2=18$ punti, quindi non ce ne sono altri.

D'altra parte, l'intersezione di $\theta \operatorname{con} \gamma_{B^{\prime}}^{\chi}$ contiene $A^{\prime}, \bar{A}^{\prime}$, e $\bar{B}^{\prime}$ con molteplicità 4, $G^{\prime}$ e $\bar{G}^{\prime}$ con molteplicità 2 ; tutti questi contano per $3 \cdot 4+2 \cdot 2=16$ punti, restano quindi 2 punti d'intersezione liberi, e ne segue che $\chi(\theta)$ possiede punti doppi in $M^{\prime \prime}=\chi\left(\gamma_{B^{\prime}}^{\chi}\right)$ e $\bar{M}^{\prime \prime}=\chi\left(\gamma_{\bar{B}^{\prime}}^{\chi}\right)$. Segue dall'osservazione fatta innanzi che i 2 rami di $\chi(\theta)$ per $M^{\prime \prime}$ avranno una tangente in comune; chiamiamola $N^{\prime \prime}$.

In fin dei conti, $\psi \circ \varphi^{-1} \circ \chi^{-1}$ appartiene al sistema lineare $D^{5}\left(M^{\prime \prime 2} \leftarrow N^{\prime \prime 2}, D^{\prime \prime 2}\right)$ e quindi abbiamo dimostrato il teorema II.

Nota. Sia $\theta$ una curva piana reale di grado $d$, con punti singolari di molteplicità $\lambda_{1}$ in $A$ e $\bar{A}, \lambda_{2}$ in $B$ e $\bar{B}, \lambda_{3}$ in $C$ e $\bar{C}$, e ancora $\lambda_{j}$ nei punti $P_{j}$ e $\bar{P}_{j}, j=4, \ldots, k$, dove i punti $A, B$ e $C$ sono come quelli considerati per i diffeomorfismi birazionali di ordine 5 e i punti $P_{j}$ sono sia in $\mathbb{P}^{2}$, sia infinitamente prossimi, ma tutti distinti da $A, B$ e $C$. I calcoli che abbiamo fatto per la prova precedente, si generalizzano per mostrare che, se $\varphi$ è un diffeomorfismo di ordine 5 corrispondente à $A, B, C$, allora

$$
\begin{aligned}
\varphi(\theta) \in D^{5 d-4\left(\lambda_{1}+\lambda_{2}+\lambda_{3}\right)} & \left(A^{\prime}\right)^{2 d-\lambda_{1}-2 \lambda_{2}-2 \lambda_{3}},\left(B^{\prime}\right)^{2 d-2 \lambda_{1}-\lambda_{2}-2 \lambda_{3}}, \\
& \left.\left(C^{\prime}\right)^{2 d-2 \lambda_{1}-2 \lambda_{2}-\lambda_{3}},\left(P_{4}^{\prime}\right)^{\lambda_{4}}, \ldots,\left(P_{k}^{\prime}\right)^{\lambda_{k}}\right)
\end{aligned}
$$

dove $A^{\prime}, B^{\prime}, C^{\prime}$ sono come precedentemente, e $P_{j}^{\prime}=\varphi\left(P_{j}\right)$. Questa è analoga alla formula delle trasformazioni di curve per trasformazioni cremoniane quadratiche nota nel caso complesso, che si trova ad esempio in [10], Ch. III,§ 2, Theorem V. 


\section{Bibliografia}

[1] M. Alberich-Carramiñana, Geometry of the Plane Cremona Maps. Lecture Notes in Math. 1769, Springer-Verlag, Berlin 2002. Zbl 0991.14008 MR 1874328

[2] A. Beauville, Variétés de Prym et jacobiennes intermédiares. Ann. Sci. École Norm. Sup. (4) 10 (1977), 309-391. Zbl 0368.14018 MR 0472843

[3] A. Corti, Factoring birational maps of threefolds after Sarkisov. J. Algebraic Geom. 4 (1995), 223-254. Zbl 0866.14007 MR 1311348

[4] F. Enriques e O. Chisini, Lezioni sulla teoria geometrica delle equazioni e funzioni algebriche (Vol. I-IV). Zanichelli, Bologna 1915-1989. Zbl 0571.51001 MR 0966664 MR 0966665

[5] R. Hartshorne, Algebraic Geometry. Grad. Texts in Math 52, Springer-Verlag, New York, Heidelberg, Berlin 1977. Zbl 0367.14001 MR 0463157

[6] H. P. Hudson, Cremona transformations in plane and space. Cambridge University Press, Cambridge 1927. JFM 53.0595.01

[7] S. Katz, The cubo-cubic transformation of $\mathbb{P}^{3}$ is very special. Math. Z. 195(1987), 255-257. Zbl 0598.14010 MR 0892055

[8] J. Kollár, Real Algebraic Surfaces. Preprint 1997, alg-geom/9/12003.

[9] K. Matsuki, Introduction to the Mori Program. Universitext, Springer Verlag, New York 2002. Zbl 0988.14007 MR 1875410

[10] J. G. Semple and L. Roth, Introduction to Algebraic Geometry. Clarendon Press, Oxford University Press, Oxford, London 1949. Zbl 0041.27903 MR 0034048

Received November 25, 2003

Felice Ronga, Université de Genève, Section de mathématiques, 2-4 rue du Lièvre, CP64, 1211 Genève 24, Suisse

E-mail: felice.ronga@math.unige.ch

Thierry Vust, Université de Genève, Section de mathématiques, 2-4 rue du Lièvre, CP64,

1211 Genève 24, Suisse

E-mail: thierry.vust@math.unige.ch 